\title{
The Complementary Roles of Feedback and Mergers in Building the Gaseous Halo and the X-Ray Corona of Milky-Way-sized Galaxies
}

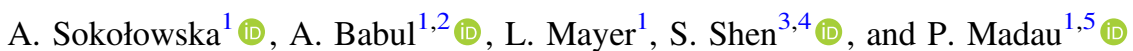 \\ ${ }^{1}$ Center for Theoretical Astrophysics and Cosmology, University of Zurich, Winterthurerstrasse 190, Zurich, Switzerland; babul@uvic.ca \\ ${ }^{2}$ Department of Physics and Astronomy, University of Victoria, Elliot Building, 3800 Finnerty Road, Victoria, Canada \\ ${ }^{3}$ Kavli Institute for Cosmology, University of Cambridge, Madingley Road, Cambridge CB3 OHA, UK \\ ${ }^{4}$ Institute of Theoretical Astrophysics, University of Oslo, Sem Saelands vei 13, Svein Rosselands hus, NO-0371 Oslo, Norway \\ 5 Department of Astronomy and Astrophysics, 1156 High Street, University of California, Santa Cruz, CA 95064, USA \\ Received 2017 August 8; revised 2018 August 9; accepted 2018 September 23; published 2018 November 1
}

\begin{abstract}
We use high-resolution cosmological hydrodynamical simulations of Milky-Way-sized galaxies with varying supernova feedback strengths and merger histories to investigate the formation of their gaseous halos and especially their hot $\left(>10^{6} \mathrm{~K}\right) \mathrm{X}$-ray-luminous coronae. Our simulations predict the presence of significant hot gas in the halos as early as $z=3-4$, well before the halos ought to be able to sustain hot mode accretion in the conventional picture. The nascent coronae grow inside-out and initially do so primarily as a result of outflows from the central galaxies powered by merger-induced shock heating and strong supernova feedback, both of which are elemental features of today's successful galaxy formation models. Furthermore, the outflows and the forming coronae also accelerate the transition from cold to hot mode accretion by contributing to the conditions for sustaining stable accretion shocks. They also disrupt the filamentary streams funneling cold gas onto the central galaxies by causing their mouths to fray into a broad delta, detach from the galaxies, and be pushed away to larger radii. And even though at early times the filaments repeatedly re-form, the hot gas and the outflows act to weaken the filaments and accelerate their ultimate disruption. Although galactic outflows are generally thought of as ejective feedback, we find that their action on the filaments suggests a preventive role as well.
\end{abstract}

Key words: galaxies: evolution - galaxies: formation - galaxies: halos - galaxies: interactions - Galaxy: halo intergalactic medium

Supporting material: animation

\section{Introduction}

Studying the formation and evolution of Milky Way (MW)like galaxies with stellar masses ranging from $3 \times 10^{10} M_{\odot}$ to $3 \times 10^{11} M_{\odot}$ has been a focus of concerted attention for several decades. These systems are ubiquitous-they compose the majority of the bright galaxies in the local universe-and they host more than two-thirds of the present-day stellar mass density (see Papovich et al. 2015 and references therein).

Although the majority of studies focus on formation and evolution of the central galaxies themselves (see, e.g., Guedes et al. 2011; Garrison-Kimmel et al. 2017; Stewart et al. 2017), one of the earliest predictions of the hierarchical structure formation models is that these galaxies ought to be cocooned by halo-filling diffuse gas, with temperatures ranging from a a few $\times 10^{5} \mathrm{~K}$ to a few $\times 10^{6} \mathrm{~K}$ (Rees \& Ostriker 1977; White \& Rees 1978).

Until about a decade ago, observational evidence for the existence of this gaseous halo was sparse and difficult to interpret (see the review on gaseous halos by Putman et al. 2012). However, significant recent progress has resulted in a compelling compendium of UV and X-ray absorption line data (Tumlinson et al. 2011; Gupta et al. 2012; Miller \& Bregman 2013; Peeples et al. 2014; Fang et al. 2015), O VII and O VIII emission line measurements (Gupta et al. 2009; Henley \& Shelton 2012, 2013; Miller \& Bregman 2015), and even the direct detection of extended X-ray emission from bright spiral galaxies more massive than the MW (Anderson \& Bregman 2011; Bogdán et al. 2013a, 2013b; Anderson et al. 2016). Nonetheless, our Galaxy is currently the only available laboratory for studying in detail the structure and the characteristics of the gaseous halos around ordinary MW-like galaxies and potentially gaining insights into their origin. The latest observational analysis indicates that the total mass of this component around the MW is $\simeq(3-5) \times 10^{10} M_{\odot}$ and extends essentially out to the virial radius (Miller \& Bregman 2015), in excellent agreement with the results from the Eris simulations discussed in Sokołowska et al. (2016).

In existing structure formation models, the origin of the gaseous halo is intimately linked to the evolution of the dark matter halos and their central galaxies. In the earlier incarnations of the galaxy formation picture, gas flowing onto a growing dark matter halo shock-heats to form a diffuse, dark matter halo-filling atmosphere. This gaseous halo was assumed to be quasi-static if its "cooling radius"- the radius where the characteristic cooling time equals the age of the universe-is close to the halo center. Otherwise, the gas within the cooling radius cools, sinks toward the halo center, and fuels both the formation of and the star formation within the central galaxy (Binney 1977; Rees \& Ostriker 1977; Silk 1977; White \& Rees 1978; White \& Frenk 1991).

A complication arises when the cooling radius exceeds the virial radius. As discussed by White \& Frenk (1991), cooling is so rapid that the infalling gas never establishes an extended quasi-static atmosphere. Although not explicitly stated at the time, this also means that a stable accretion shock cannot form at the virial radius, and the infalling gas will flow onto the galaxy without experiencing an accretion shock. Based on their shock stability analysis, Birnboim \& Dekel (2003) identified the critical halo mass at which a stable shock can be maintained 
at the virial radius to be $\simeq 10^{11}-10^{12} M_{\odot}$, depending on the metallicity of the gas.

The above picture and the calculations are, however, rooted in the idealized, spherical collapse, and spherical halo models. In practice, both the accretion history and the flow geometry of the mass converging onto a halo are much more complex. In lockstep with the emergence of virialized structure, the largescale matter distribution in the universe organizes into a network of filaments and sheets, and as the virialized structures grow hierarchically, the filaments also merge and grow in scale (Cautun et al. 2014). Initially, the halos of MW-like systems form at the intersections of filaments, but eventually they are incorporated into either larger filaments (field galaxies) or larger virialized systems (group and cluster galaxies).

Successful execution of increasingly realistic, high-resolution galaxy formation simulations within their proper cosmological setting has radically transformed our understanding of how baryons accrete onto galactic halos and how galaxy formation proceeds. For one, the bulk of the gas accreting onto a halo at a redshift $0 \leqslant z \lesssim 3$ is never heated to the halo's virial temperature if the halo mass is less than $3 \times 10^{11} M_{\odot}$. Fardal et al. 2001; Katz et al. 2003; Kereš et al. 2005, 2009; Brooks et al. 2009; Dekel et al. 2009). In fact, the simulations suggest two distinct modes of smooth gas accretion onto the galactic halos: (i) "cold mode" accretion, where the gas accretes via "cold" filamentary streams that often penetrate to the halo centers, and (ii) "hot mode" accretion, where the accreting gas encounters a stable accretion shock and the combination of this and the subsequent compressive heating raises its temperature to from $T \sim$ a few $\times 10^{5}$ to as much as a few $\times 10^{6} \mathrm{~K}$ (see Kereš et al. 2005, 2009; Dekel \& Birnboim 2006; Dekel et al. 2009).

In this revised paradigm, initially the gas enters the galactic halos through narrow, cold, dense filamentary streams. As the streams converge onto the central galaxy, they experience compressive heating, but while the gas cooling time is shorter than the heating time, the streams remain cold and deposit their gas directly onto the central galaxy (van de Voort et al. 2011; Stewart et al. 2017). Eventually, the cooling efficiency of the gas drops, the cooling time exceeds the compressive heating time, and an increasing fraction of the gas starts to form the galaxy's diffuse gaseous halo instead of settling into the galaxy (Joung et al. 2012). The emergence of this atmosphere sets the stage for a stable accretion shock at the virial radius.

Concurrently, on the supragalactic scale, the cosmic filaments are growing, and as their widths become comparable to the sizes of the halos, an increasing fraction of the gas flows onto the halos in quasi-spherical fashion, shock heating as it encounters the accretion shock, and then heating up further as it is squeezed deeper into the halo. It is this gas that is generally thought to build the diffuse gaseous halos (van de Voort et al. 2011).

Before proceeding, we emphasize that most of the discussion of hot and cold mode accretion in the literature refers to the accretion onto the central galaxy. Here, we are mainly interested in gas accretion onto the galactic halos ${ }^{6}$ as it pertains to the establishment of the gaseous halo and especially the $\mathrm{X}$-ray-luminous corona. We also caution that the simple picture sketched out here should be viewed as a conceptual aide. In

\footnotetext{
6 For a thorough discussion of the similarities and difference between gas flow onto the halo and gas flow onto the galaxy, we refer the reader to van de Voort et al. (2011).
}

practice, the different stages do not unfold sequentially, and there are times when both the hot and the cold modes coexist. In general, the timing of the transition from predominantly cold to largely hot mode accretion depends on each halo's individual growth rate, but for MW-like galaxies, it typically occurs around $z \sim 2$ (Kereš et al. 2009).

The studies of Fardal et al. (2001), Katz et al. (2003), Kereš et al. (2005, 2009), and Dekel \& Birnboim (2006) that laid the foundation for the above picture, though groundbreaking, did not include the full set of subgrid implementations of the key physical processes that gird today's successful galaxy formation simulations. The simulations of Kereš et al. (2005, 2009), for example, lack sufficiently effective supernova (SN) feedback required to prevent the overproduction of stars. Contemporary studies have shown that strong SN feedback, in concert with other stellar feedback processes like stellar winds, radiation pressure, and photoheating, is crucial for (i) obtaining realistic stellar and baryonic masses and star formation efficiencies in halos with masses as high as $10^{13} M_{\odot}$ (Davé et al. 2011, 2012; Christensen et al. 2016; Liang et al. 2016), (ii) ensuring that the lower-mass end of the galaxy stellar mass function agrees with observations (e.g., Davé et al. 2011; Bower et al. 2012; Puchwein \& Springel 2013), and (iii) realizing disk galaxies with reasonable global and local properties across a broad mass range: dwarfs (Shen et al. 2014), spiral galaxies (Guedes et al. 2011; Marinacci et al. 2014; Roškar et al. 2014; Agertz \& Kravtsov 2015; Garrison-Kimmel et al. 2017), and massive early-type galaxies (Fiacconi et al. 2014).

A common feature of all the simulations is that strong SN feedback engenders powerful galactic outflows (see the review by Somerville \& Davé 2015, and references therein). This is not a surprise; numerous studies dating back to Mathews \& Baker (1971), Larson (1974), Dekel \& Silk (1986), and Babul \& Rees (1992) have anticipated this. Moreover, it seems inevitable that the expulsion of a large fraction of the gas out of the galaxies and into the halo (and beyond) ought to have a profound impact on the emergence and evolution of the gaseous halo. This was hinted at by White \& Frenk (1991), and several simulation-based studies (e.g., Oppenheimer \& Davé 2006; Davé et al. 2011, 2012; van de Voort et al. 2011, 2016; Christensen et al. 2016; Stewart et al. 2017) of galaxy formation have also commented on this, noting that galactic outflows are required to account for the metallicity gradients within the gaseous halos, that they tend to make the gaseous halos more radially extended-in the Eris simulation of the MW galaxy, the total baryonic mass fraction within the virial radius is only $70 \%$ of the universal value (Sokołowska et al. 2016) - and that both the size and the mass of these halos are correlated with the star formation episodes (van de Voort et al. 2016).

In this paper, we present a detailed investigation of the origin of the gaseous halo around MW-like galaxies. We pay particular attention to the roles of the different accretion modes, merger-induced shocks (see also Sinha \& HolleyBockelmann 2009), and SN feedback in building the gaseous halo. The present study is motivated by our previous paper (Sokołowska et al. 2016, hereafter Paper I), where we explored the present-day diffuse halos of MW-like galaxies using a suite of high-resolution, zoomed-in hydrodynamic simulations. Our principal simulation, Eris, employs an SN feedback prescription that is remarkably successful at making a realistic MW-like 
galaxy (Guedes et al. 2011). In Paper I, we showed that the key properties of this virtual MW's gaseous halo are also in excellent agreement with recent observational constraints for our Galaxy's halo, namely, an X-ray luminosity in the $0.5-2 \mathrm{keV}$ band of $\sim 10^{39} \mathrm{erg} \mathrm{s}^{-1}$, coronal density sufficient to ram pressure strip MW satellites, and a radial electron density profile consistent with that implied by the O VII absorption line measurements of Miller \& Bregman (2013) (see Sokołowska et al. 2016, for details). We also identified an intriguing feature in the halo of our simulated galaxy: a central spheroidal region with a radial extent of $100-140 \mathrm{kpc}\left(0.6 r_{\mathrm{vir}}\right)$ embedded in a $T=10^{5}-10^{6} \mathrm{~K}$ warm-hot atmosphere containing most of the mass. Within this central region (hereafter referred to as the corona), the density of the hot $\left(T>10^{6} \mathrm{~K}\right)$ gas exceeds that of the warm-hot gas.

Here, we examine the buildup of the corona and the gaseous halo not only in Eris but also, for comparison, in (i) ErisNFB, i.e., Eris with SN feedback switched off, (ii) Eris $2 k$ (Sokołowska et al. 2016, 2017), a variant with the same initial conditions (IC) as Eris but with enhanced cooling as well as boosted SN feedback (see also Shen et al. 2012, 2013), and (iii) Venus (Sokołowska et al. 2017), a new addition to our inventory of zoomed-in simulations of MW-like galaxies with a more active merger history than Eris.

The present paper is organized as follows: In Section 2, we motivate why we investigate this particular set of runs and describe the physics included in the simulations. In Section 3, we present our results about the emergence of the gaseous halos, including the inside-out growth of the hot corona, in Eris and Venus simulations. We discuss the roles of stellar feedback and merger-induced shocks in giving rise to the structure seen in our simulations, and we explore the connection between the growth of the corona and the inflowing cold filamentary network. This is then followed by a summary and concluding remarks in Section 4.

\section{Simulations}

Our study is based first on two unique, high-resolution, cosmological, zoom-in simulations of MW-sized spiral galaxies, Eris and Venus. The simulations assume the flat WMAP-3 cosmology $\left(\Omega_{M}=0.24, \Omega_{b}=0.042, H_{O}=73 \mathrm{~km} \mathrm{~s}^{-1} \mathrm{Mpc}^{-1}\right.$, $\left.n=0.96, \sigma_{8}=0.76\right)$ and were performed with the tree-smoothed particle hydrodynamics (SPH) code GASOLINE (Wadsley et al. 2004) with mass resolution $m_{\mathrm{dm}} \simeq 9.8 \times 10^{4} M_{\odot}$ and $m_{\mathrm{SPH}} \simeq$ $2 \times 10^{4} M_{\odot}$ and spatial resolution $\simeq 120 \mathrm{pc}$.

The late-time spiral galaxy in the first of the two runs, Eris, has been previously shown to be an excellent match to the MW (Guedes et al. 2011). The second simulation, Venus, was run with the same subgrid prescriptions as Eris but employs different IC while ensuring that the final virial halo mass $\left(\sim 8 \times 10^{11} M_{\odot}\right)$ and the halo spin parameter $(\lambda \sim 0.03)$ are nearly identical to that in Eris. As a result, although the galaxies in Eris and Venus both form at the intersection of four dark matter filaments, their mass convergence pattern is very different (see Figure 1). The dominant halo in Eris assembles early on and has a relatively quiet merging history, with the last major $^{7}$ merger occurring at $z=3.1$. On the other hand, Venus has a much more active merger history and experiences twice as many major mergers as Eris, with the last major merger occurring at $z=0.9$. Also, multiple progenitors of

Defined as a merger with mass ratio $>0.1$ between the two galaxies. comparable mass evolve separately in Venus for a long time, with the single dominant halo and its associated galaxy only appearing after the last major merger. The amount of substructure at $z=0$ is also more abundant in Venus than in Eris, both in the stellar and in the dark matter components. In particular, a large satellite orbits around the primary galaxy in Venus at late times, exciting perturbations in the disk of the central galaxy at pericenter passages as late as $z=0.24$.

As for the physical processes, both simulations include radiative and Compton cooling. The radiative cooling is computed for a simple mixture of $\mathrm{H}$ and $\mathrm{He}$ via nonequilibrium cooling rates in the presence of the ionizing cosmic ultraviolet (UV; Haardt \& Madau 1996) background (Wadsley et al. 2004). Additionally, $T<10^{4} \mathrm{~K}$ gas can cool through fine structure and metastable lines of $\mathrm{C}, \mathrm{N}, \mathrm{O}, \mathrm{Fe}, \mathrm{S}$, and $\mathrm{Si}$ (Bromm et al. 2001; Mashchenko et al. 2007).

Both runs utilize the same star formation prescriptions (see Stinson et al. 2006, for details). Briefly, the gas particles must have a density greater than the star formation threshold of $n_{\mathrm{SF}}=5$ atoms $\mathrm{cm}^{-3}$ and temperature $T<10^{4} \mathrm{~K}$ in order to form stars. Particles that satisfy these requirements are stochastically selected to form stars according to $d M_{*} / d t=c^{*} M_{\mathrm{gas}} / t_{\mathrm{dyn}}$, where $M_{*}$ is the mass of stars created, $c^{*}$ is a constant star formation efficiency factor (set to 0.1 in all runs), $M_{\text {gas }}$ is the mass of gas creating the star, and $t_{\mathrm{dyn}}$ is the gas dynamical time. Each star particle then represents a population of stars, covering the entire initial mass function (IMF). We adopt the Kroupa et al. (1993) IMF, and stars more massive than $8 M_{\odot}$ are assumed to explode as Type II SNe (SNe II) at the end of their lives.

In both runs, Type I SNe (SNe I) and SNe II enrich the interstellar medium (ISM) with metals and inject energy into the medium. According to the "blast wave feedback" model of Stinson et al. (2006), SN energy is introduced as purely thermal injection of energy, as the blast wave shocks are expected to convert the kinetic energy of ejecta into thermal energy on scales smaller than those resolved by our simulations. Once energy is introduced (the fraction of SN energy that couples to the interstellar medium is $\epsilon_{\mathrm{SN}}=0.8$ ), the particles receiving the energy are prevented from cooling for typically 10-50 Myr, with the cooling shut-off timescale being computed as the sum of the Sedov-Taylor (Taylor 1950; Sedov 1959) and snowplow phases in the ejecta (McKee \& Ostriker 1977). By delaying the cooling, we model, in a phenomenological way, the unresolved effects of momentum driving and heating by turbulent dissipation in the ejecta before they reach the radiative phase. Delaying the cooling also prevents artificial overcooling of gas heated by SN feedback. The strength of feedback depends on the number of SNe produced, which is in turn governed by the IMF and, locally, by the star formation density threshold.

Apart from Eris and Venus, we also consider two additional simulations, both variants of Eris: ErisNFB and Eris $2 k$. Both runs have identical IC to Eris. ErisNFB has the same physics as Eris, except that the SNe do not inject any energy into the ISM (see Section 3.3), while in Eris2k (see Section 3.5) the physics set is augmented to allow gas at $T>10^{4} \mathrm{~K}$ to cool via metalline radiation and more efficient metal-line cooling of gas at $T<10^{4} \mathrm{~K}$ (see Shen et al. 2012, 2013), SN feedback is boosted, and turbulent diffusion of thermal energy and metals is enabled following Shen et al. (2010). Metal-line cooling is computed with the code CLOUDY (Ferland et al. 1998) under the assumption that the metals are in ionization equilibrium 


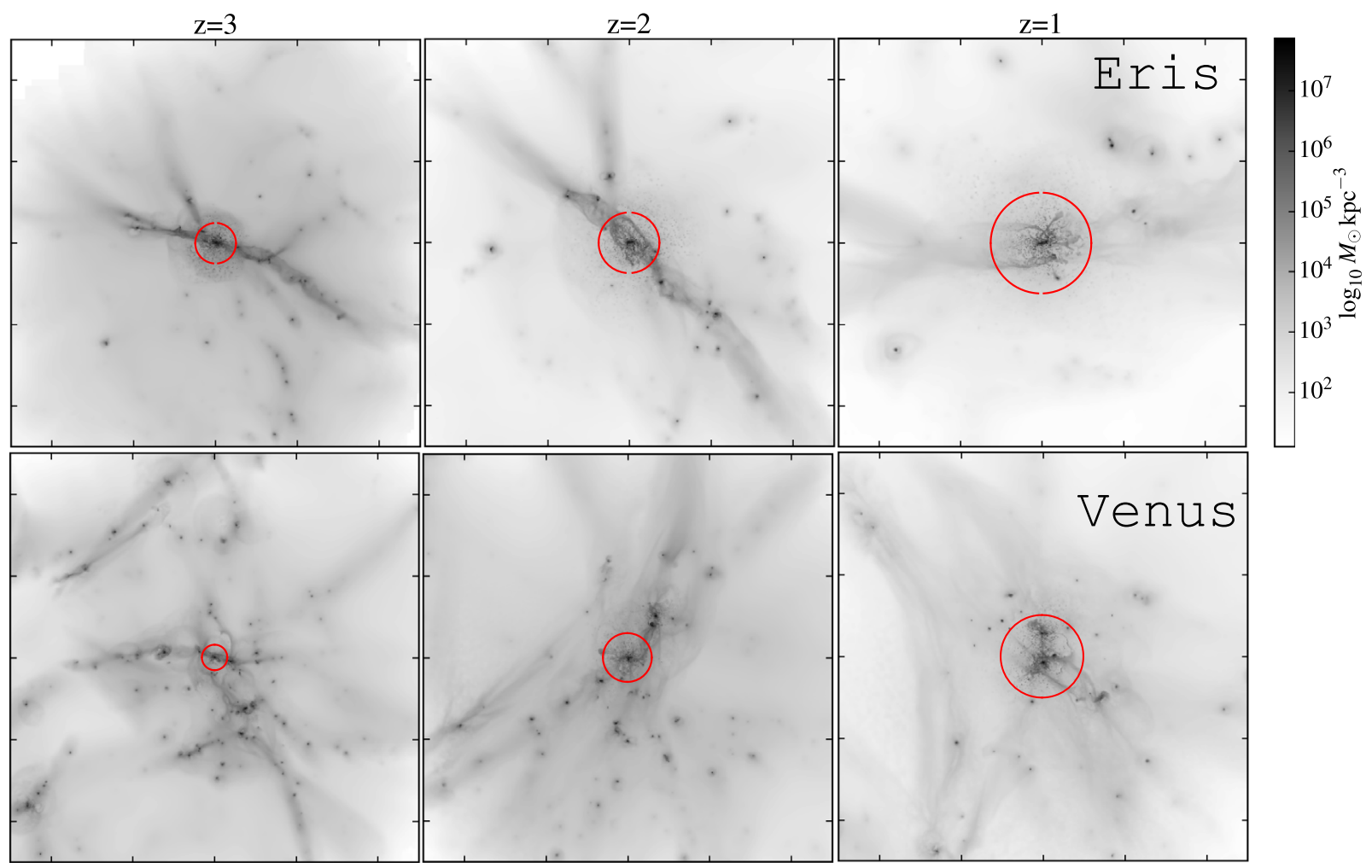

Figure 1. Density maps of gas around the galaxies (shown edge-on) in the two simulations under consideration: Eris (quiet accretion history) and Venus (active accretion history). The width of each square is 1 comoving Mpc. The cross sections of the most massive progenitor halo at each time step are shown as red circles.

(Shen et al. 2010), in the presence of an updated cosmic ionizing background (Haardt \& Madau 2012). SN feedback is enhanced both locally and globally by simultaneously raising the star formation density threshold to 100 atoms $\mathrm{cm}^{-3}$ (see Guedes et al. 2011; Hopkins et al. 2012; Mayer 2012 for a discussion about the resulting local enhancement of SN feedback) and adopting a more top-heavy Kroupa (2001) IMF than the Kroupa et al. (1993) IMF used in Eris, which yields about a factor of 2.8 more $\mathrm{SNe}$ at the same star formation rate. We refer interested readers to Sokołowska et al. (2016, 2017) for more details about the Eris $2 k$ simulation. Table 1 summarizes the runs discussed in this paper.

\section{Results}

In Paper I, we discussed in detail the properties and the structure of the low-redshift gaseous halo in the Eris simulation. We found that this halo consists of a central spherical region about the primary galaxy, containing predominantly hot $\left(T>10^{6} \mathrm{~K}\right)$, and surrounded by an atmosphere for warm-hot $\left(10^{5}-10^{6} \mathrm{~K}\right)$ gas extending out to the virial radius. We labeled the central region the "hot corona" and will refer to it as such in the present paper too. The temperature of the hot gas within the corona is a factor of $\sim 2-2.5$ greater than the system's virial temperature at $z=0$ (Balogh et al. 1999; van de Voort et al. 2011) of

$$
T_{\text {vir }} \approx 4 \times 10^{5} \mathrm{~K}\left[\frac{M_{\text {halo }}(z)}{7.6 \times 10^{11} M_{\odot}}\right]^{2 / 3}(1+z),
$$

where we have scaled the relationship to Eris's dark matter halo mass at $z=0$. The corona has a radius of about $100 \mathrm{kpc}$ at $z=0.5$ and grows to $140 \mathrm{kpc}$ at $z=0$, equivalent to $0.6 r_{\mathrm{vir}}$. Its
Table 1

Input Parameters of the Runs

\begin{tabular}{lccccccc}
\hline \hline Run & UVB & IMF & $n_{\mathrm{SF}}$ & $T_{\max }$ & $\epsilon_{\mathrm{SN}}$ & MC & IC \\
\hline Eris & HM96 & K93 & 5 & $3 \times 10^{4}$ & 0.8 & low-T & Q \\
Eris2k & HM12 & K01 & 100 & $1 \times 10^{4}$ & 1.0 & all-T & Q \\
Venus & HM96 & K93 & 5 & $3 \times 10^{4}$ & 0.8 & low-T & A \\
\hline
\end{tabular}

Note. Notation: UVB — UV background (HM96: Haardt \& Madau 1996; HM12: Haardt \& Madau 2012); IMF—initial mass function (K93: Kroupa et al. 1993; K01: Kroupa 2001); $n_{\mathrm{SF}}$-star formation density threshold in $\mathrm{cm}^{-3}$; $T_{\max }$-maximum temperature (in K) of gas particles that can participate in star formation; $\epsilon_{\mathrm{SN}}-\mathrm{SN}$ efficiency parameter in $10^{51} \mathrm{erg} ; \mathrm{MC}-$ metal cooling; and IC—initial conditions (Q: quiet merger history; A: active merger history).

mass is approximately $7 \times 10^{9} M_{\odot}$, while that of the warm-hot gas is about four times larger, i.e., $\sim 3 \times 10^{10} M_{\odot}$. The combined properties of the hot corona and the warm-hot atmosphere in Eris are in excellent agreement with a number of different observational constraints and characteristics of the MW halo gas, including its electron density profile and the $\mathrm{X}$-ray luminosity of $\sim 10^{39} \mathrm{erg} \mathrm{s}^{-1}$ in the $0.5-2 \mathrm{keV}$ band (see Sokołowska et al. 2016, and references therein for details).

Here, we start by assessing Venus. As we did with Eris, we compute the X-ray luminosity of the gas particles within the virial radius of a halo using the radiative rates from the Astrophysical Plasma Emission Code3 (APEC; Smith et al. 2001), which assumes an optically thin gas in collisional ionization equilibrium (for more details on the calculation, see Paper I). We find that the X-ray luminosity of Venus, $L_{\mathrm{X}}=1.2 \times 10^{39} \mathrm{erg} \mathrm{s}^{-1}$, is also in excellent agreement with the MW observations. 


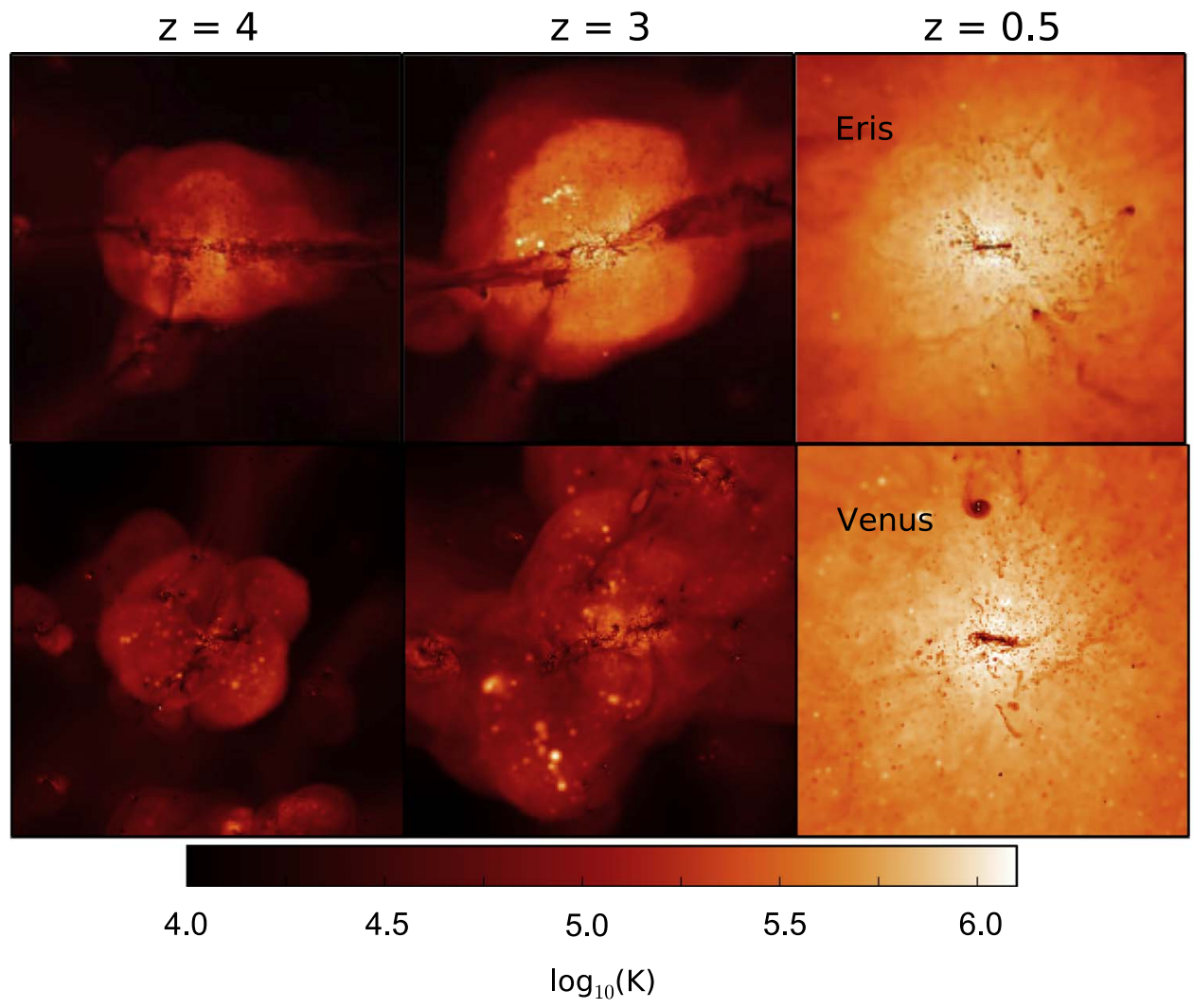

Figure 2. Two pathways to the same result: a galaxy surrounded by an X-ray-bright corona. Temperature maps of the gaseous halo of Venus and Eris centered on the most massive progenitor halo, at three different times. The width of each square is 300 comoving kpc. Compare with Figure 1 of Sokołowska et al. (2016).

Figure 2 compares the Eris and Venus gas temperature maps at three different time steps: $z=4,3$, and 0.5. As mentioned previously, the configuration of the filamentary network in the two simulations is different, resulting in two distinct evolutionary sequences for the gas. For example, at $z=4$, cold filamentary flows penetrate the halos of the main progenitors in both runs, but gas in Eris is hotter. At $z=3$, a central region containing both hot and warm-hot gas has formed about the most massive progenitor galaxy in Eris, while in Venus the gas has yet to heat up to the same temperatures and is also more widely distributed among the multiple progenitors. By $z=0.5$, however, Venus acquires a corona/gaseous halo like that in Eris.

That both Eris and Venus reach similar endpoints with respect to the structure of gaseous halo/corona, even though they do so via different evolutionary pathways, raises a number of questions regarding the processes that govern their evolution and facilitate the convergence at $z=0$. In what follows, we analyze the simulations to identify the key processes involved. The one phenomenon that stands out is powerful episodic galactic outflows. We also discuss the impact of the outflows and the growing hot coronae on other structures in the galactic halo, including the cold filaments depositing gas onto the central galaxy, and on the transition from cold filamentary to hot quasi-spherical accretion mode.

Throughout this paper, we follow the schema adopted in Paper I and discuss the distribution and the properties of gas in different temperature ranges, namely, cold gas $(T<3 \times$ $\left.10^{4} \mathrm{~K}\right)$, warm gas $\left(3 \times 10^{4} \mathrm{~K}<T<10^{5} \mathrm{~K}\right)$, warm-hot gas $\left(T=10^{5}-10^{6} \mathrm{~K}\right)$, and hot gas $\left(T>10^{6} \mathrm{~K}\right)$. We do so to facilitate comparison with the observational literature where the discussion is informed by the diagnostics used to study the gaseous halos (see the review by Putman et al. 2012, for details). For completeness, we note that the first two categories correspond to gas probed via $\mathrm{H} \mathrm{I}$ and $\mathrm{H} \alpha$ emission lines, as well as by various low and intermediate ion absorption lines, including Ly $\alpha, \mathrm{Mg}$ II, S III, S IIII, C II, C III, and O I. The third category corresponds to gas studied via $\mathrm{CIV}$ and $\mathrm{O}$ VI absorption lines, and the fourth corresponds to X-ray emissions and $\mathrm{O}$ VII and $\mathrm{O}$ VIII lines.

\subsection{Growth of the Diffuse Medium: An Overview}

The conventional description (see, e.g., Kereš et al. 2009) of the formation of the diffuse gaseous halos and their X-rayluminous coronae assumes that they are primarily generated by hot mode accretion onto the halos once the halos' mass exceeds the critical mass threshold, that is, the accreting gas is shockheated by a stable accretion shock at the virial radius. In the post-shock region, the gas that enters at an earlier time is pushed deeper into the halo by currently inflowing gas. If the cooling time for this diffuse gas is longer than the compression time, the gas heats up as it is squeezed deeper into the halo. Joung et al. (2012) find that in their simulated MW-like galaxies the dominant component of this inward-flowing diffuse gas is $<10^{5} \mathrm{~K}$ beyond the virial radius, $10^{5} \mathrm{~K}<$ $T<10^{6} \mathrm{~K}$ (i.e., warm-hot) down to $\approx 50 \mathrm{kpc}$, and then $>10^{6} \mathrm{~K}$ (i.e., hot) within the innermost $20-50 \mathrm{kpc}$. Here, we assess this picture in the context of our simulations.

In panel (a) of Figure 3, we show the evolving total (virial) halo mass for the most massive progenitor halo in Eris and Venus as a function of redshift. The gray band shows the Dekel \& Birnboim (2006) metallicity-dependent critical mass threshold that they argue a halo must grow to in order to sustain 
a)

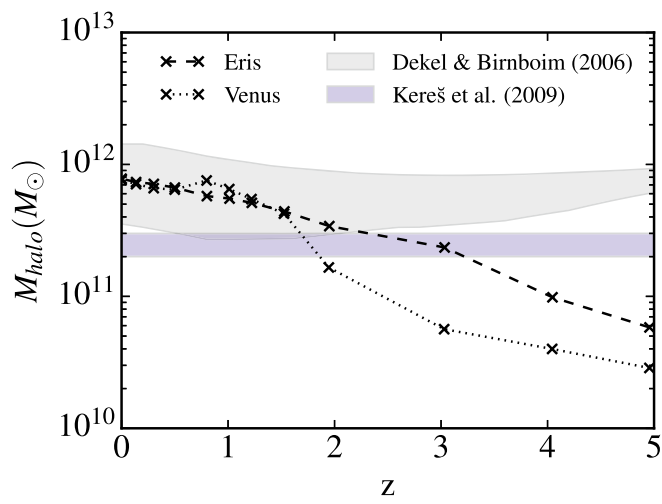

b)

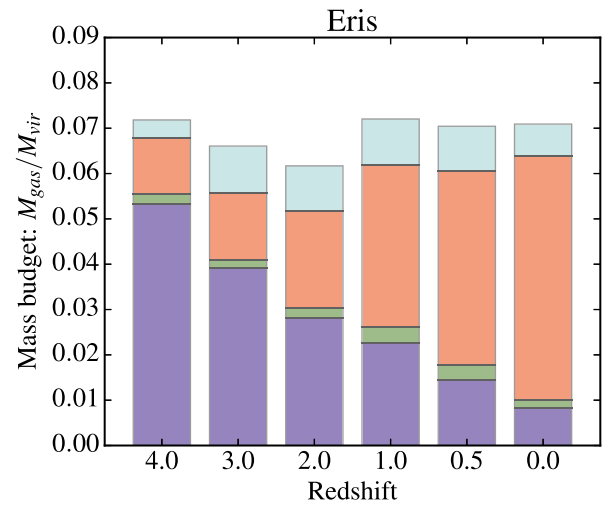

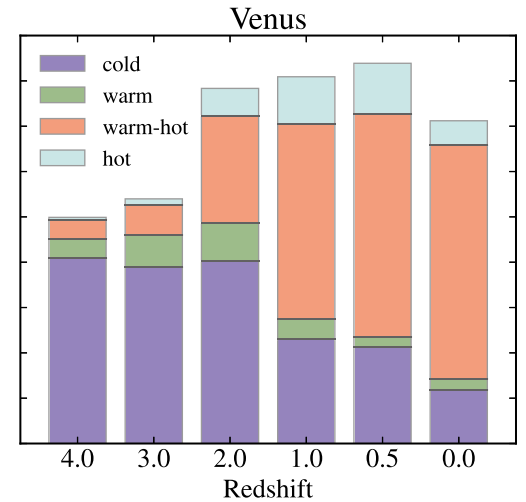

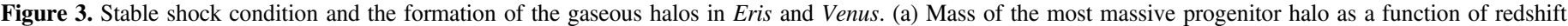

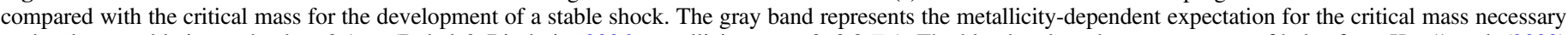

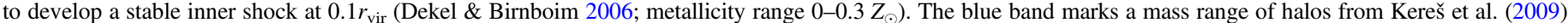

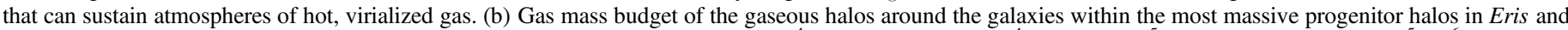

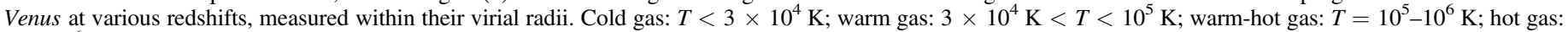
$T>10^{6} \mathrm{~K}$.

an inner shock at $0.1 r_{\text {vir. }}$. The band's width is due to its dependence on metallicity and corresponds to a spread of $0.03-0.3 Z_{\odot}$. In this model, the critical mass for sustaining a stable virial shock (at $r_{\text {vir }}$ ) exceeds $10^{12} M_{\odot}$, even with $Z=0.03 Z_{\odot}$, and hence sits above the gray band. The blue band corresponds to the nearly redshift-independent (at least for $0 \leqslant z \leqslant 3$ ) threshold mass range of $(2-3) \times 10^{11} M_{\odot}$, where halos start to develop diffuse gaseous atmospheres in the simulations of Kereš et al. (2009). This is also the mass range where the accretion onto the halos transitions from predominantly cold to predominantly hot mode. Given their different mass accretion histories, it is not surprising that Eris and Venus simulations transition to the hot mode accretiondominated phase at different times. Eris crosses into the Kereš et al. (2009) blue band at $z=3$ and into the gray band at $z=2$. Venus lags Eris, crossing the blue and gray bands at $z=2$ and $z=1.5$, respectively.

In panels (b) of Figure 3, we show the gas mass budget within the virial radius of the most massive progenitor halos of Eris (left) and Venus (right) at six different redshifts. The gas mass is normalized to the virial mass of the halo at the redshifts under consideration and categorized by temperature: cold $\left(T<3 \times 10^{4} \mathrm{~K}\right)$, warm $\left(3 \times 10^{4} \mathrm{~K}<T<10^{5} \mathrm{~K}\right)$, warm-hot $\left(T=10^{5}-10^{6} \mathrm{~K}\right)$, and hot $\left(T>10^{6} \mathrm{~K}\right)$. We explicitly exclude gas in the galactic disk, i.e., gas with density higher than the star formation density threshold (5 atoms/cc), in order to isolate the gaseous halo. The differences in the gas fractions between the two simulations are due to their different assembly history, i.e., the relatively low gas fraction before $z=2$ in Venus is correlated with its late halo assembly. However, after Venus undergoes its last major merger and the halo masses in the two simulations converge, the gas fractions in both runs become similar too.

Examining the distribution of gas across the different phases in Eris first, we find that although the cold gas (blue) is the most abundant component until $z=2$, there is already significant warm-hot, and especially hot, halo gas by $z=3$. The two components combined compose $\simeq 30 \%$ of the total diffuse gas budget at $z=4$ and $\simeq 40 \%$ at $z=3$. In Venus the hot and warm-hot diffuse gas composes $\simeq 20 \%$ at $z=3$ and $\simeq 40 \%$ by $z=2$. Of particular interest to us is the presence of the nearly constant fraction of hot $\left(>10^{6}\right)$ gas at $0.5 \lesssim z \lesssim 3$ in
Eris and at $0.5 \lesssim z<2$ in Venus. This fraction is $14 \%-16 \%$ in Eris and 12\%-13\% in Venus. The temperature of this gas is a factor of $\sim 2-3$ higher than the virial temperature of the halos at these epochs: $T_{\mathrm{vir}}=(5.3,7.5,7.1,6.8,6.1) \times 10^{5} \mathrm{~K}$ for Eris and $(2.9,2.8,4.5,7.4,6.0) \times 10^{5} \mathrm{~K}$ for Venus at $z=$ $(4,3,2,1,0.5)$, respectively. Although this hot gas is not the most abundant gas phase, it is primarily responsible for the extended diffuse X-ray emissions and is also the component that is traced by the $\mathrm{O}$ VII/O VIII absorption/emission. As we summarized in the Introduction and discussed in much greater detail in Paper I, the low- $z$ hot gas properties in our simulations agree remarkably well with the observations.

To identify the origin of the hot gas, we flag the gas particles within the virial radius that compose this component at $z=0$ and track their temperatures back in time. We also note the time when they accreted onto the most massive progenitor halo in the two simulations. We treat a particle as accreted when it crosses the virial radius of the halo for the first time.

In Figure 4, we show that the gas that ends up in hot coronae at $z=0$ is not accreted exclusively at late epochs. A significant fraction (i.e., $\sim 30 \%$ ) of it is accreted at early times, over the first 5-6 Gyr, and is cold at the time. This cold-accreted gas, which comes in via the filamentary streams and is, early on, primarily deposited onto the central galaxy, must be heated to over a million degrees and expelled from the galaxy in order to become a part of a hot corona. There are only two main mechanisms that can do this: strong shock heating and dispersal of the gas as a result of major mergers (Poole et al. 2006, 2008; Sinha \& Holley-Bockelmann 2009) and/or heating and expulsion via SN-driven galactic outflows (e.g., Oppenheimer \& Davé 2006; Davé et al. 2011, 2012; van de Voort et al. 2011, 2016; Christensen et al. 2016; Stewart et al. 2017). We note that we write "and/or" because mergers, especially gas-rich mergers, are frequently associated with starbursts.

The contribution of the cold-accreted gas to the coronae drops off steeply after $\approx 5.5 \mathrm{Gyr}$ of evolution (i.e., after $z \sim 1.15$ ) in favor of warm and warm-hot accretion in both simulations. By this time, both halos are of similar mass and growing at nearly the same rate. The warm/warm-hot accretion flow never settles onto the central galaxy, and the fraction that contributes to the corona is gas that is initially shock-heated 

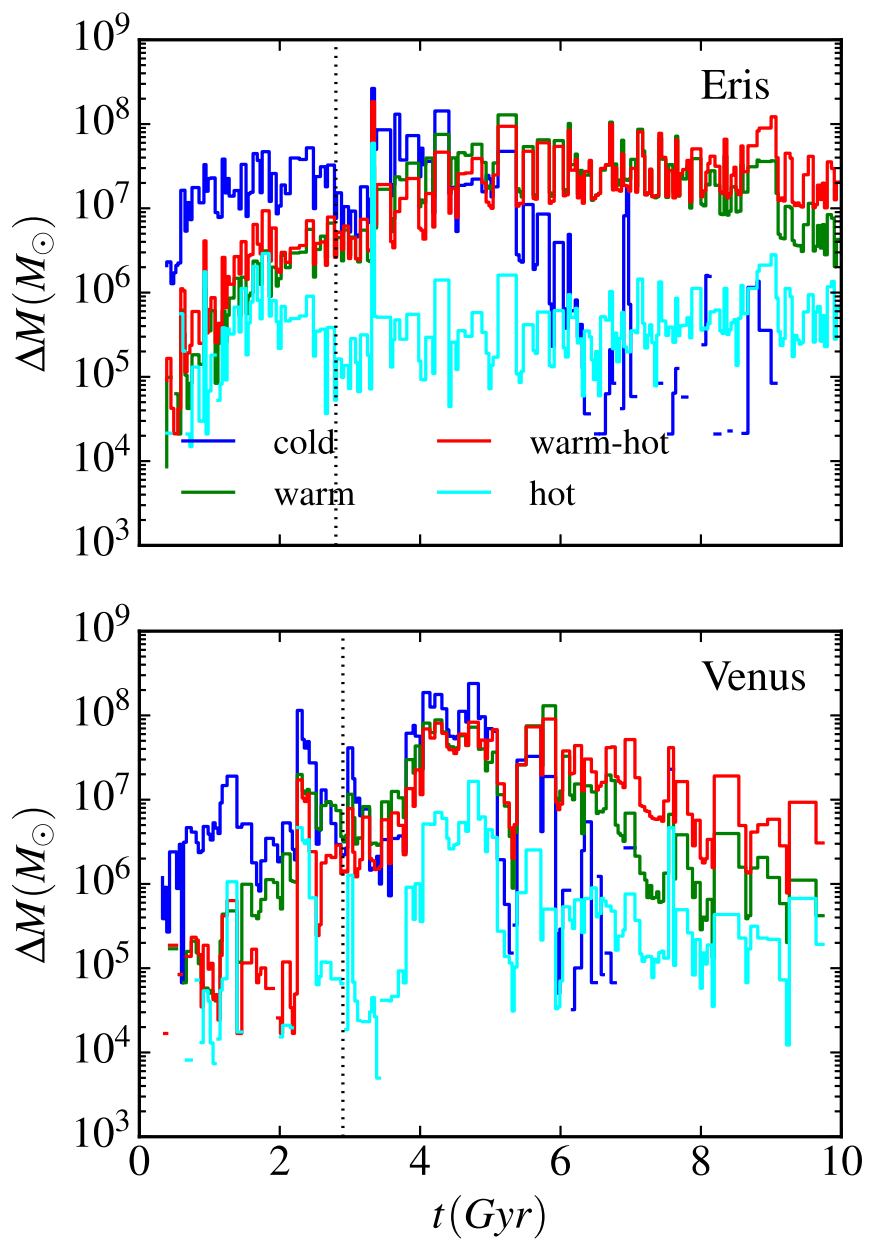

Figure 4. Temperature of gas particles present in a $z=0$ hot corona at first $r_{\mathrm{vir}}$ crossing, showing two-stage halo gas evolution. The dotted line marks approximately the time of the equivalence of the cold and hot mode accretion $(z=2.5)$.

upon accretion and then heated to coronal temperatures either via compression or via additional shocks in the inner halo owing to interactions with the outflowing winds.

Figure 4 also shows the time when the amount of coldaccreted gas first equals the amount of gas coming in via warmhot and hot modes. This is delineated by the black dotted line (and referred to as the epoch of "equivalence") and corresponds to approximately $z=2.5$ for Eris and $z=2.3$ for Venus. We did not expect that these times would be comparable given that the mass of the dominant halos in the two runs is different by a factor of a few at the time (see Figure 3(a)). However, from about $z=2.9$ to 1.4 , Venus experiences a series of gas-rich mergers. Each merger leads to a sharp increase in the diffuse gas fraction, resulting in an overall accelerated growth to a similar level to that in Eris by $z=2$ (panel (b) of Figure 3).

\subsection{The Distribution and Thermal Properties of the Gaseous Halo}

In this subsection, we investigate the distribution of diffuse gas that composes the gaseous halo and characterize its thermal evolution as a function of time. As previously, we ignore the gas that belongs to the disk, i.e., all the gas with density higher than the star formation density threshold of 5 atoms/cc.

First, we present the temperature profiles of the gaseous halo in Figure 5. This is the mass-weighted average temperature of
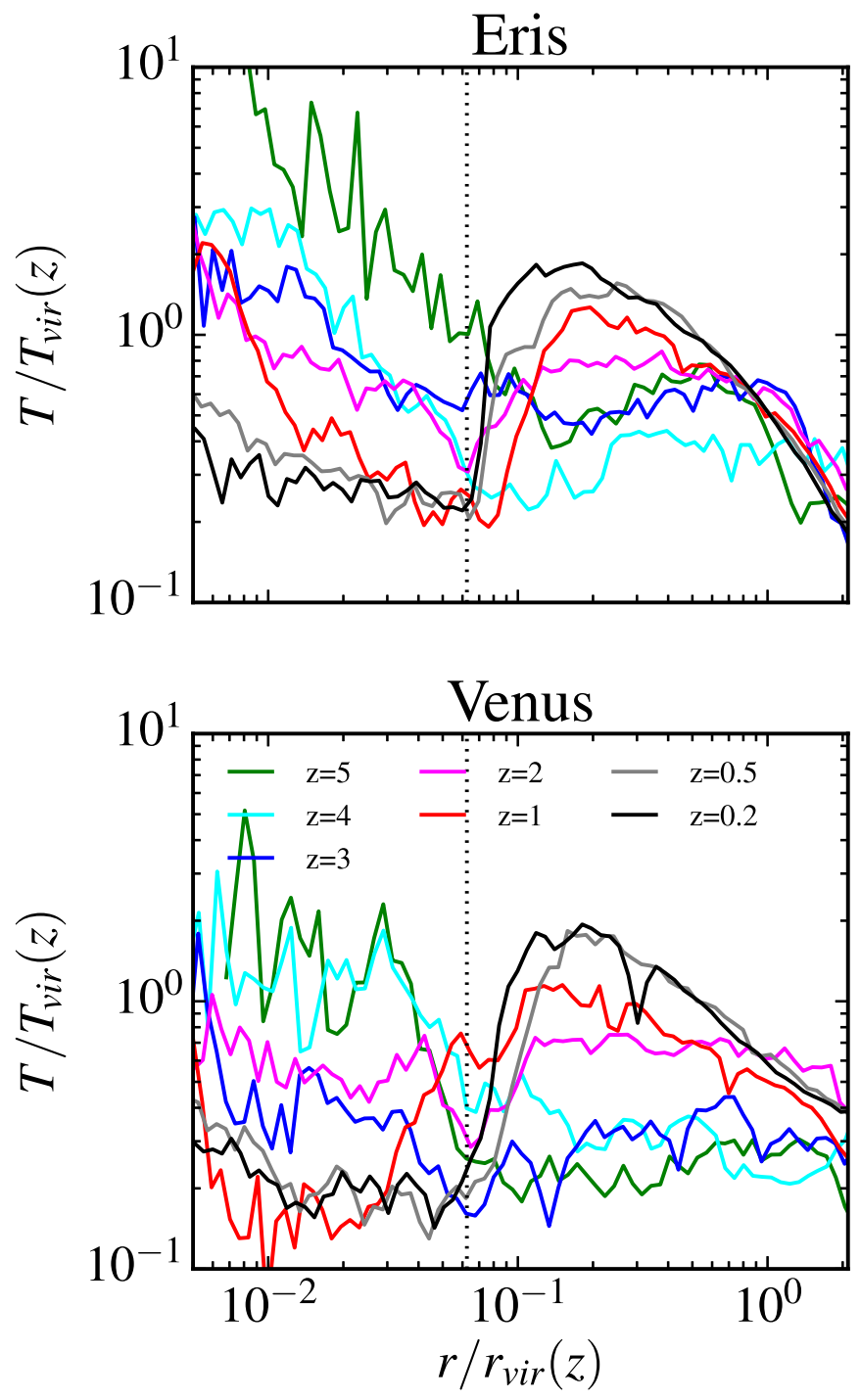

Figure 5. Radial distributions of gas temperature normalized to the virial temperature of the halo at a given redshift, showing the diffuse halo in formation. The vertical line marks the approximate extent of the disk at $z=0$. Top: Eris; bottom: Venus.

the gas in radial shells around the galactic center and normalized to the virial temperature of the halo: $T_{\text {vir }}=(5.3$, $7.5,7.1,6.8,6.1) \times 10^{5} \mathrm{~K}$ for Eris and $(2.9,2.8,4.5,7.4$, $6.0) \times 10^{5} \mathrm{~K}$ for Venus at $z=(4,3,2,1,0.5)$, respectively. One can distinguish two regions in these profiles, to the right and left of the black vertical line $\left(r / r_{\text {vir }}=0.06\right)$, which marks the approximate extent of the galactic disk at $z=0$. The gas to the left cools to a fraction of the virial temperature and flows into and supplies fresh gas to the galactic disk. The gas to the right grows hotter with time. Prior to $z \approx 2$, the gas is nearly isothermal across this region, but thereafter the temperature distribution has a maximum near $0.2 r_{\mathrm{vir}}(40 \mathrm{kpc}$ at $z \approx 0)$ and declines to $\simeq 0.5 T_{\text {vir }}(z)$ at the virial radius. Such an outwardly declining temperature profile is a normal feature of the hierarchical structure formation models (see, e.g., Lewis et al. 2000). At late times, the peak temperature increases to approximately twice the virial temperature. The particular shape of $T / T_{\text {vir }}$ suggests that some heating process (or a combination thereof) localized to the inner halo or related to the galactic disk is responsible for the hot corona, which, as we 

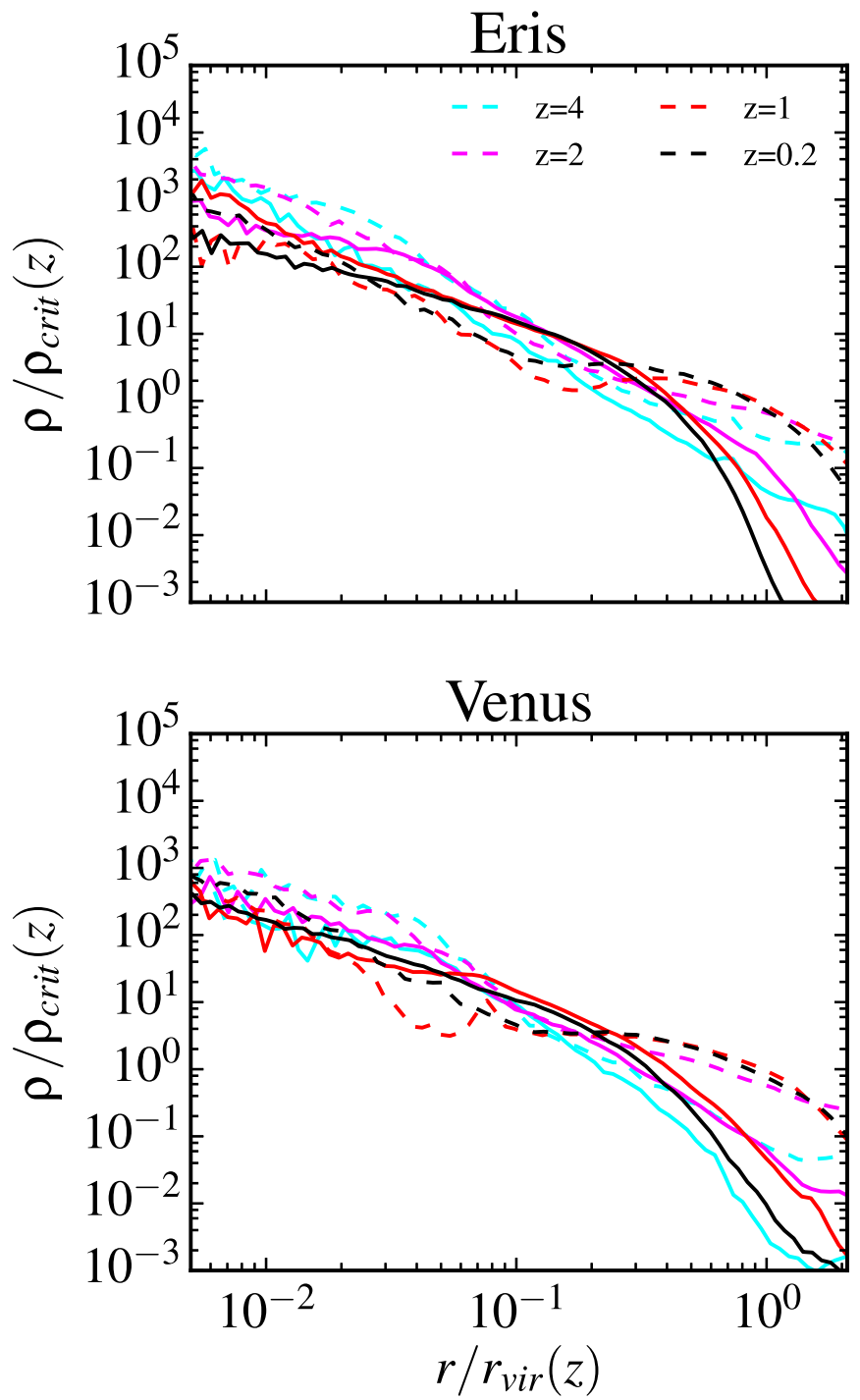

Figure 6. Radial density distribution of gas and its evolution with redshift. Dashed and solid lines denote the warm-hot and hot components of gas, respectively. Quantities are normalized to the critical density and the virial radius of the galaxy at a given redshift and show the development of the onionlike structure: a corona embedded in the warm-hot soup of gas.

have mentioned previously, cocoons the galaxy and extends out to $0.6 r_{\text {vir }}$ at low redshifts.

Next, we look specifically at the spatial distributions of the hot $\left(>10^{6} \mathrm{~K}\right)$ and warm-hot $\left(10^{5}-10^{6} \mathrm{~K}\right)$ components of the gaseous halos. In Figure 6, we compare their density profiles at different redshifts. At first $(z=4)$, warm-hot and hot gas profiles in both simulations fall off as a power law with increasing radii, and the density of the warm-hot gas is higher. After $z=2$, however, several differences arise: First, although the power-law index of the warm-hot gas profile at small radii remains approximately unchanged, its amplitude decreases. It also develops a shallow trough with a minimum at $(0.1-0.2) r_{\text {vir }}$ and then rises slightly to a local maximum before dropping off at large radii. At the same time, the hot gas profile flattens slightly to $0.4 r_{\text {vir }}$ before dropping off steeply. This steep dropoff indicates that hot gas can be found only within a fraction of a virial radius $\left(\sim(0.6-0.8) r_{\text {vir }}\right)$ and not beyond. Additionally, the evolution of the two profiles jointly results in a pocket between $\sim 0.05 r_{\text {vir }}$ and $\sim 0.5 r_{\text {vir }}$ (Eris) and between $\sim 0.02 r_{\text {vir }}$

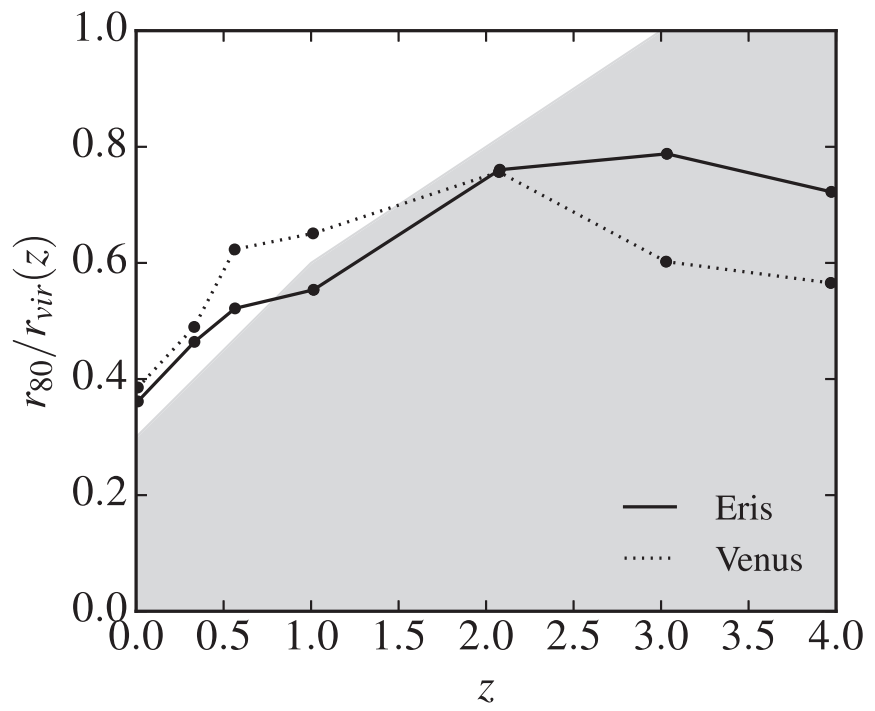

Figure 7. Size evolution of the hot gas halo. $r_{80}$, defined as a radius encompassing $80 \%$ of its mass within the virial radius, is normalized to the virial radius $\left(r_{\text {vir }}\right)$. The gray shaded region indicates where cooling of hot gas is still important (i.e., $t_{\text {cool }} \ll t_{\text {Hubble }}$ ).

and $\sim 0.2 r_{\text {vir }}$ (Venus) where the hot gas concentration exceeds that of the warm-hot gas. This pocket emerges at $z \simeq 2$ in Eris.

We quantify the extent of the hot gas as a function of time in Figure 7 . We define its extent as a radius encompassing $80 \%$ of the total hot gas mass within the virial radius $\left(r_{80}\right)$ and find that before the formation time of the corona (i.e., around $z=4-3$ ), the hot gas extends out to $80 \%$ of the virial radius. As the halo accretion mode switches to hot mode, the hot gas component in the halos is increasingly confined by the accumulating blanket of warm-hot gas, to $0.6 r_{\text {vir }}$ at $z=2$ and decreasing with time to $0.4 r_{\text {vir }}$ by $z=0$.

The gray shaded area in Figure 7 shows the radius within which the cooling time of the hot gas is shorter than the Hubble time, as a function of the redshift. To estimate this cooling radius, we calculated cooling time as a function of radius according to $t_{\text {cool }}=U / \Lambda$, where both the internal energy, $U$, and the radiative cooling rate, $\Lambda$, are taken from the simulations. We have verified that in both simulations the cooling time is very short near the galactic center (e.g., within $0.1 r_{\text {vir }}$ it drops to orders of magnitude below 1 Gyr even at $z=1$ ) and monotonically increases with radius. Nonetheless, the cooling time of all of the hot gas within the virial radius at $z=3$ is less than $1 \mathrm{Gyr}$ and that of all the hot gas within, for example, $0.8 r_{\text {vir }}$ at $z=2,0.6 r_{\text {vir }}$ at $z=1$, and $0.3 r_{\text {vir }}$ at $z=0$ is less than the Hubble time. This means that there should not be any hot gas in the central regions unless it was either being continually replenished or reheated. Shock heating of the gas as a result of major mergers or heating/replenishment by galactic outflows would both do the trick.

\subsection{Role of Feedback and Mergers}

In this section, we attempt to identify the major heating mechanisms that contribute to the formation and buildup of the hot corona. The design of our simulation code, GASOLINE, allows us to determine the impact of each of the following heating mechanisms on the diffuse halo gas: radiative heating $\left(\dot{E}_{\mathrm{rad}}\right)$, SN thermal feedback $\left(\dot{E}_{\mathrm{SN}}\right)$, and artificial viscosity $\left(\dot{E}_{\mathrm{AV}}\right)$, which reflects heating due to shocks, mixing, etc. Here "shocks" refers not only to accretion shocks but also to shocks 

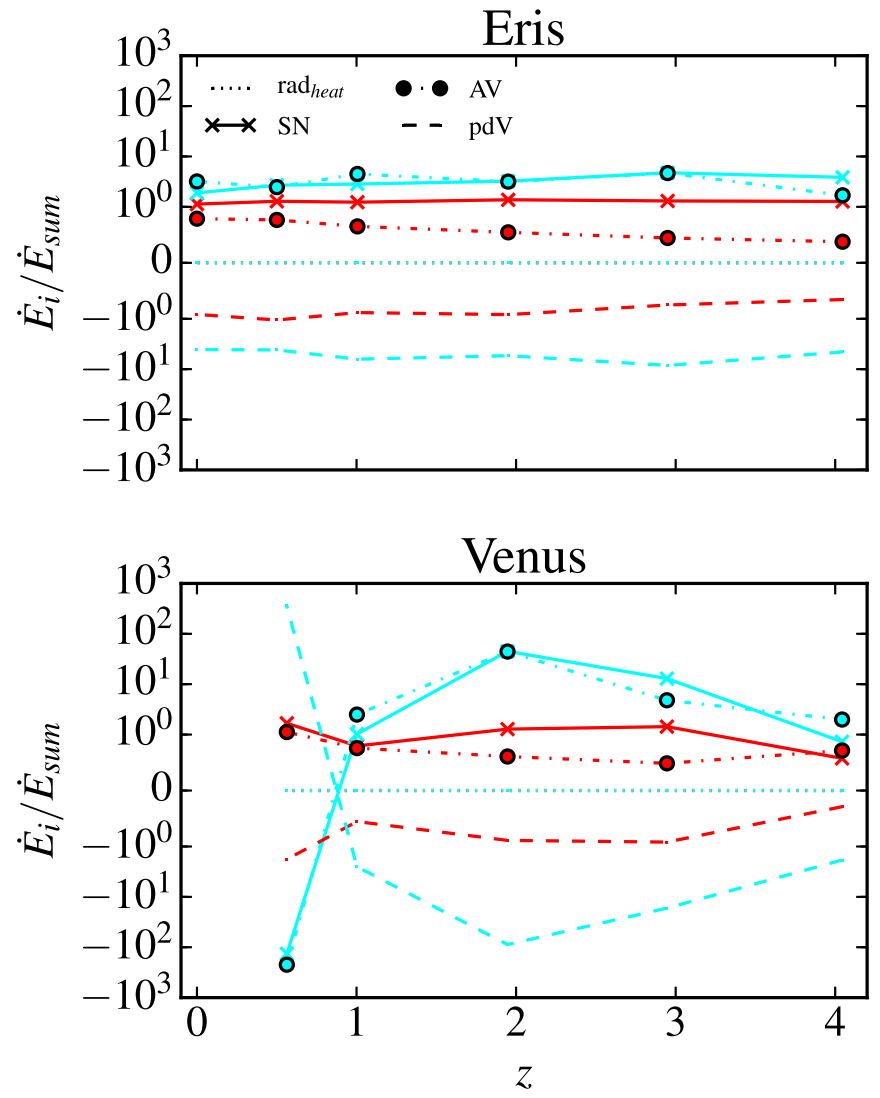

Figure 8. Energy injection rates for various gas phases in the region encompassed by a sphere of a virial radius around a galaxy, normalized to the total heating rate. The color coding corresponds to the warm-hot (red) and hot (cyan) gas phases. Legend: $\operatorname{rad}_{\text {heat }}$-heating due to atomic/radiative processes only; $\mathrm{SN}-\mathrm{SN}$ thermal feedback; AV—artificial viscosity; pdV—work done by/on the gas.

in general, including those triggered by mergers, galactic outflows, etc. We also show the heating (cooling) due to adiabatic compression (expansion) $\left(\dot{E}_{\mathrm{pdV}}\right)$. While the first three $\dot{E}_{i}$ are non-negative, the latter can be positive (indicating heating due to compression) or negative (indicating cooling due to expansion).

We focus on two particular phases of gas: the warm-hot gas $\left(T=10^{5}-10^{6} \mathrm{~K}\right)$ and the hot gas $\left(T>10^{6} \mathrm{~K}\right)$. We compute the mass-weighted $\dot{E}_{i}$ for the different heating mechanisms for the gas within the virial radius as a function of redshift and normalize all energy injection rates to the total heating rate $\dot{E}_{\text {sum }}=\sum_{i} \dot{E}_{i}$, where $i$ stands for each of the processes mentioned above.

The red and cyan lines in Figure 8 show the results for the warm-hot and hot gas, respectively. To start with, we note that the impact of the radiative heating on the two phases is negligible. We also find that both phases are expanding (and hence cooling) at all redshifts. This is true even at the lowest redshift in Venus despite the dashed cyan line's sudden steep rise to the positive domain. As we have mentioned previously, Venus undergoes a major merger at $z=0.9$, and in the aftermath the hot gas expands and cools so rapidly that even the total energy injection rate $\dot{E}_{\text {sum }}$ becomes negative, and consequently $\dot{E}_{\mathrm{SN}} / \dot{E}_{\text {sum }}, \dot{E}_{\mathrm{pdV}} / \dot{E}_{\text {sum }}$, and $\dot{E}_{\mathrm{AV}} / \dot{E}_{\text {sum }}$ all change signs. As for the heating mechanisms, Figure 8 shows that both SN heating and shocks/mixing contribute equally to the heating of the hot phase across all redshifts, while $\mathrm{SN}$ heating is slightly more important for the warm-hot gas. We reiterate that shock heating is not exclusively due to shocks associated with gravitational processes (i.e., accretion and mergers) but also includes contributions from shocks engendered by galactic outflows. In other words, $\dot{E}_{\mathrm{SN}}$ represents the minimum amount of energy injected by the SNe. Taken together, the above findings show that $\mathrm{SN}$ heating and subsequent inside-out expansion play a pivotal role in the buildup of the hot corona and the warm-hot phase in our MW-like systems.

Yet another feature that points to the importance of feedback and galactic outflows is the high metallicity of the warm-hot and the hot diffuse gas from as early as $z=7$. Figure 9 shows the radial metallicity profiles (in solar units assuming $Z_{\odot}=0.0194$ (Anders \& Grevesse 1989)) for different phases of gas at three epochs, $z=7, z=3$, and $z=0.5$, in the Eris simulation. The trends in Venus are very similar. As in the previous section, we are interested in the metallicity of the diffuse gas only and not in the disk; therefore, we only consider gas below the star formation threshold density. The results show that the hot and the warm-hot phases are the most and the second-most metal-rich phases, respectively, with a nearly constant metallicity, within the range $0.3-0.5 Z_{\odot}$ for the hot phase and $0.1-0.2 Z_{\odot}$ for the warm-hot phase, at least as far out as $2 r_{\text {vir }}$ and as early as $z=7$. The metals in our simulations come from $\mathrm{SNe}$ I and $\mathrm{SNe}$ II within galaxies, and galactic outflows are the only available mechanism for transporting these out into the halo and beyond (see Davé et al. 2012; Rahmati et al. 2016). The difference in the metallicity of the hot and warm-hot gas is the result of the latter being a mixture of high-metallicity galactic outflows and cosmological influx of low-metallicity gas that accretes onto the halo. We also note that there is an inward rise in the profiles developing close to the halo center at $z=3$, which suggests that the metal-rich outflows are increasingly confined at late times as the potential well deepens and the gaseous halo itself grows.

To further understand the origin of the warm-hot and hot gas in the diffuse halo, we divide the gaseous halo in Eris into three zones: (i) disk-dominated ( $r_{0}<15 \mathrm{kpc}$ ), (ii) corona-dominated $\left(r_{0} \in(15,100) \mathrm{kpc}\right)$, and (iii) the outer warm-hot reservoir $\left(r_{0} \in(100,240) \mathrm{kpc}\right)$. We determine the fraction of gas mass in the different phases in each zone. The results are shown in Table 2. We then select 100 particles from each zone, distributed across the different phases in accordance with the mass fractions (e.g., the representative set of particles for the central region comprises 90 cold, 1 warm, 4 warm-hot, and 5 hot particles), color-code the particles by their $z=0$ phase, track them back in time, and follow their evolution forward in time on the radius-temperature $(r-T)$ diagram. Two such diagrams, for $z=2.72$ (left column) and 0.54 (right column), are shown in Figure 10, where we indicate the typical trajectories of particles with black arrows; an animation (movie) illustrating the time evolution in a more visually informative format is included with the supplementary material.

This experiment allowed us to distinguish three different patterns of accretion and their evolution. These can be categorized into two types, with the left column in Figure 10 being an example of the first type and the right column being an example of the second type.

We first consider the innermost zone. Not surprisingly, the gas enters the halo via cold flows at early times and flows onto the central galaxy. At late times, the gas enters the halo and is 

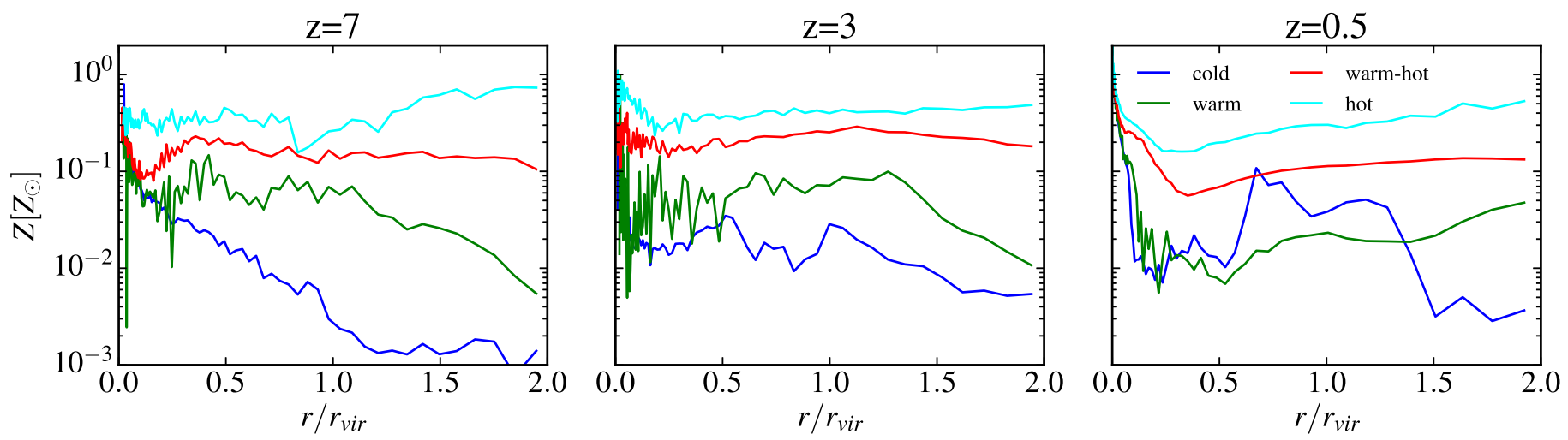

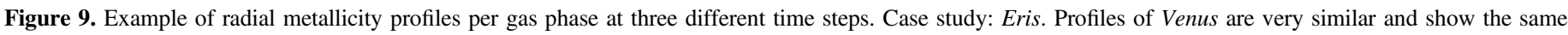
trend: hot gas is the most metal-enriched gas phase, and warm-hot gas is the second-most metal-enriched gas phase.

Table 2

Mass Fraction of Gas in the Four Phases in Each of the Three Zones, at $z=0$

\begin{tabular}{lcccc}
\hline \hline Zone & Cold & Warm & Warm-hot & Hot \\
\hline$r_{0}<15 \mathrm{kpc}$ & $90 \%$ & $1 \%$ & $4 \%$ & $5 \%$ \\
$r_{0} \in(15,100) \mathrm{kpc}$ & $9 \%$ & $0 \%$ & $58 \%$ & $33 \%$ \\
$r_{0} \in(100,240) \mathrm{kpc}$ & $2 \%$ & $3 \%$ & $92 \%$ & $3 \%$ \\
\hline
\end{tabular}

shock-heated to $T \sim 10^{5} \mathrm{~K}$ close to the virial radius but rapidly cools down to below $3 \times 10^{4} \mathrm{~K}$. The gas that ends up in this central zone, for the most part, remains there, and even when feedback dramatically increases the temperature of some of the particles, their evolution is more akin to a fountain. They cool down very rapidly and return to the central zone.

The middle zone (second panel in each column) is built by circulation flows. At early times, the gas enters the virial radius through cold flows and ends up in the galaxy. Subsequently, it is heated by $\mathrm{SN}$-driven or merger-induced shocks and expelled from the galaxy. Some of the gas directly contributes to the hot and warm-hot gas in the second zone, while the rest flows out of the halo and then falls back in and is shock-heated upon reaccretion. Around $z=1.5$, the pattern changes. The incoming gas follows two different paths in the $r-T$ diagram: some of the gas follows the trajectory of the gas that ends up in zone 1, except that this gas is subsequently heated and expelled from the inner halo, while the rest is heated via accretion shocks to $T>10^{5} \mathrm{~K}$ and flows directly into the middle zone. That both hot mode accretion and cold mode accretion exist at the same time is expected while the halos are transitioning from predominantly cold to predominantly hot (Kereš et al. 2009; van de Voort et al. 2011). A significant fraction of the gas that composes the hot corona is that which has been heated, and often driven outward, by shocks occurring within the galaxy or deep in the inner halo.

Finally, the gas in the outermost zone (third panel) initially comes in primarily as cold flows, is heated and expelled from the galaxy, and expands to fill the outer zone. Some of this gas expands beyond the virial radius and eventually falls back in. While expanding, the gas cools and drops below the $10^{6} \mathrm{~K}$ threshold. This is the clearest indication that a fraction of the outer envelope of warm-hot gas in fact started out as hot gas. Around $z=1.9$, this inflow-to-outflow channel is overtaken by a different pathway: the gas is heated just prior to crossing the virial radius. This gas receives no SN energy; accretion shock is the primary heating agent. However, a fraction of the accreting gas still cools, flows into the galaxy, and returns as outflow.

Next, we quantify the difference that the presence of SN feedback makes on the diffuse gas budget and its evolution. In order to do so, we perform a direct test of the impact of SN feedback on the halo, namely, we run another simulation (hereafter ErisNFB), which is identical to Eris in every aspect except that the SN feedback is switched off after $z=4$. This redshift was chosen as a compromise involving various competing concerns: (i) the simulation completes in a reasonable period of time, ${ }^{8}$ (ii) the diffuse gaseous halo should ideally be absent, and (iii) the lack of feedback should have little or no influence on the assembly history. Although one cannot produce realistic galaxies without feedback, the benefit of this test lies in being able to isolate the effect of SN heating from gravitational heating by accretion shocks and merger-induced shocks. We note that although we do the above experiment with Eris, we expect similar results for Venus.

Figure 11 illustrates a violent heating episode, which is an example of a series of such events occurring repeatedly over the lifetime of our Eris run, and compares the magnitude of this event in the original Eris run with feedback (top row) and in ErisNFB (bottom row). The temperature maps in Figure 11 capture the evolution of the "blast" over $0.3 \mathrm{Gyr}$. In both Eris and ErisNFB, the general sequence is similar: at $z=3.42$, the gaseous halo consists of two bubbles of warm-hot gas that are separated by inflows of cold/warm gas. By $z=3.26$, the central concentration of over one-million-degree gas grows significantly into a nearly spherical region approximately $20 \mathrm{kpc}$ in radius. Thereafter, the hot gas expands and cools. Early "blasts" in Eris are easy to detect in the temperature maps, and their timing coincides with peaks in the star formation rate.

The second row of Figure 11 shows that the hot gas can also be generated much earlier than expected even in the absence of feedback. As early as $z=3.42$, gas in the center of ErisNFB exceeds the temperature threshold of $10^{6} \mathrm{~K}$. Here the outflows are generated by shock heating associated with a major merger and can be seen in the map as conical warm-hot and hot patches extending beyond the virial radius of the halo at that epoch. However, the outflows are weaker and contain less hot gas (by mass) than those generated in Eris, where SN II feedback acts in concert with the merger. The extent to which

\footnotetext{
8 Simulations that allow for cooling but no feedback tend to slow down dramatically as cold gas builds up.
} 

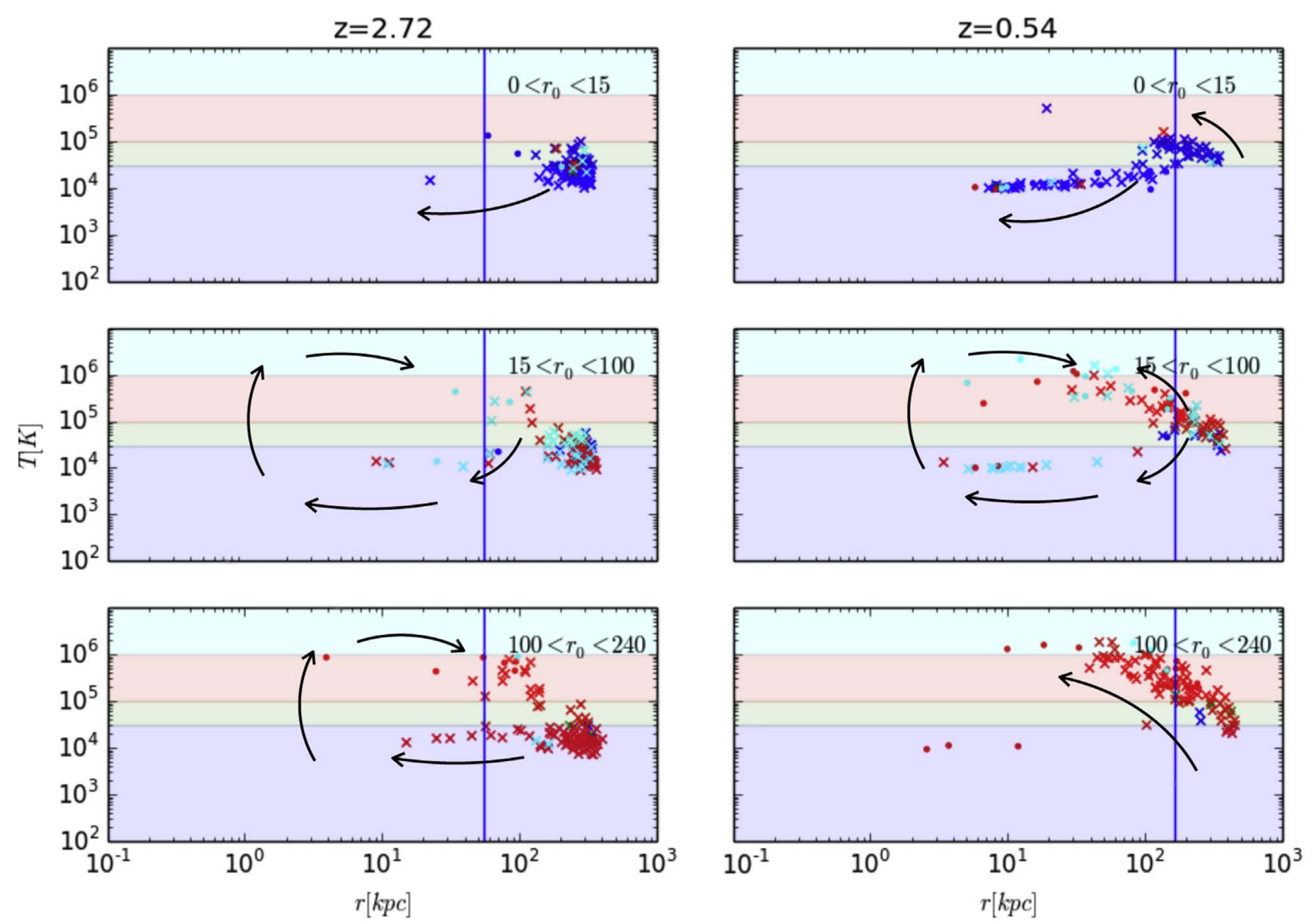

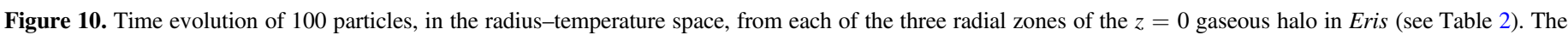

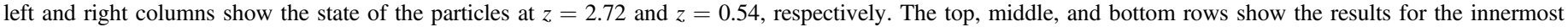

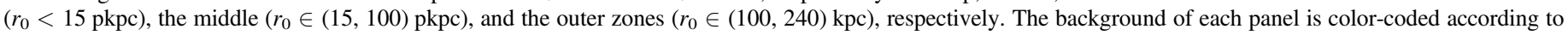

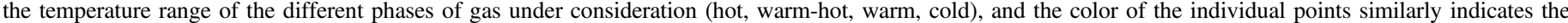

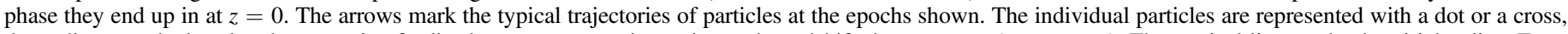

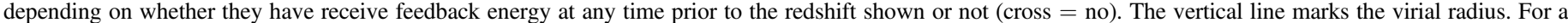
detailed description of the experiment, including the selection method, see the text. Case study: Eris.

(An animation of this figure is available.)

the heated gas expands by $z=2.99$ in ErisNFB compared to Eris (bottom right panel vs. the top right panel of Figure 11) attests to this.

In Figure 12, we show the radial density profiles of the two gas phases: warm-hot (top) and hot (bottom) for the run with SN feedback (solid lines) and without (dashed lines) at $z=3$ and $z=2$. The absence of feedback results in significant differences in the inner region of the diffuse gaseous halo $\left(\lesssim 0.3 r_{\text {vir }}\right)$, particularly with respect to the warm-hot phase. The density of the warm-hot gas in that region is almost two orders of magnitude higher if $\mathrm{SN}$ feedback is switched on. The difference between the density of hot gas in Eris and ErisNFB is slightly smaller but no less significant, namely, hot gas is about five times denser in Eris than in ErisNFB. By $\sim 0.6 r_{\mathrm{vir}}$, the density profiles of both gas phases converge. In terms of mass, we find 1.3 times more hot gas in Eris than in ErisNFB within the virial radius at $z=3$, and by $z=1$, that ratio increases slightly to 1.5 . In total, introducing feedback increased the abundance of (warm-hot + hot) $T>10^{5} \mathrm{~K}$ gas within the virial radius by a factor of 1.3-1.5 between $z=3$ and 1 .

The gaseous halos in Eris and ErisNFB not only are structurally different but also result in divergent $\mathrm{X}$-ray evolution with redshift. We compute X-ray luminosities at $z=3,2$, and 1 in the $0.5-2 \mathrm{keV}$ band for Eris and ErisNFB using the same procedures as described in the start of Section 3. We find that the X-ray luminosities are higher when feedback is included, namely, $3.40 \times 10^{41}, 8.9 \times 10^{40}$, and $5.9 \times 10^{40} \mathrm{erg} \mathrm{s}^{-1}$ as opposed to $2.4 \times 10^{38}, 1.1 \times 10^{38}$, and $1.3 \times 10^{37} \mathrm{erg} \mathrm{s}^{-1}$ at $z=3,2$, and 1 , respectively. In the absence of feedback, the gaseous halo is considerably underluminous and in fact fails to match the observed X-ray luminosity.

The above result is the product of three effects. In the nofeedback run, the amount of hot gas expelled out of the galaxy is lower to start with, and this gas tends to cool down faster. Both of these lead to a smaller, less massive corona and a lower X-ray luminosity (see Figure 11, as well as Toft et al. 2002). Additionally, as illustrated in Figure 12, the run with SN feedback has a considerably higher density of hot (and warmhot) gas within the inner $\sim 0.6 r_{\text {vir }}$. Since the X-ray emissivity scales as $\rho_{\text {gas }}^{2}$, this makes for a significantly brighter inner halo. SN feedback is, therefore, essential for obtaining not only realistic disk galaxies but also realistic diffuse halos.

Before continuing, we would be remiss if we did not point out that our findings about the importance and impact of heated 


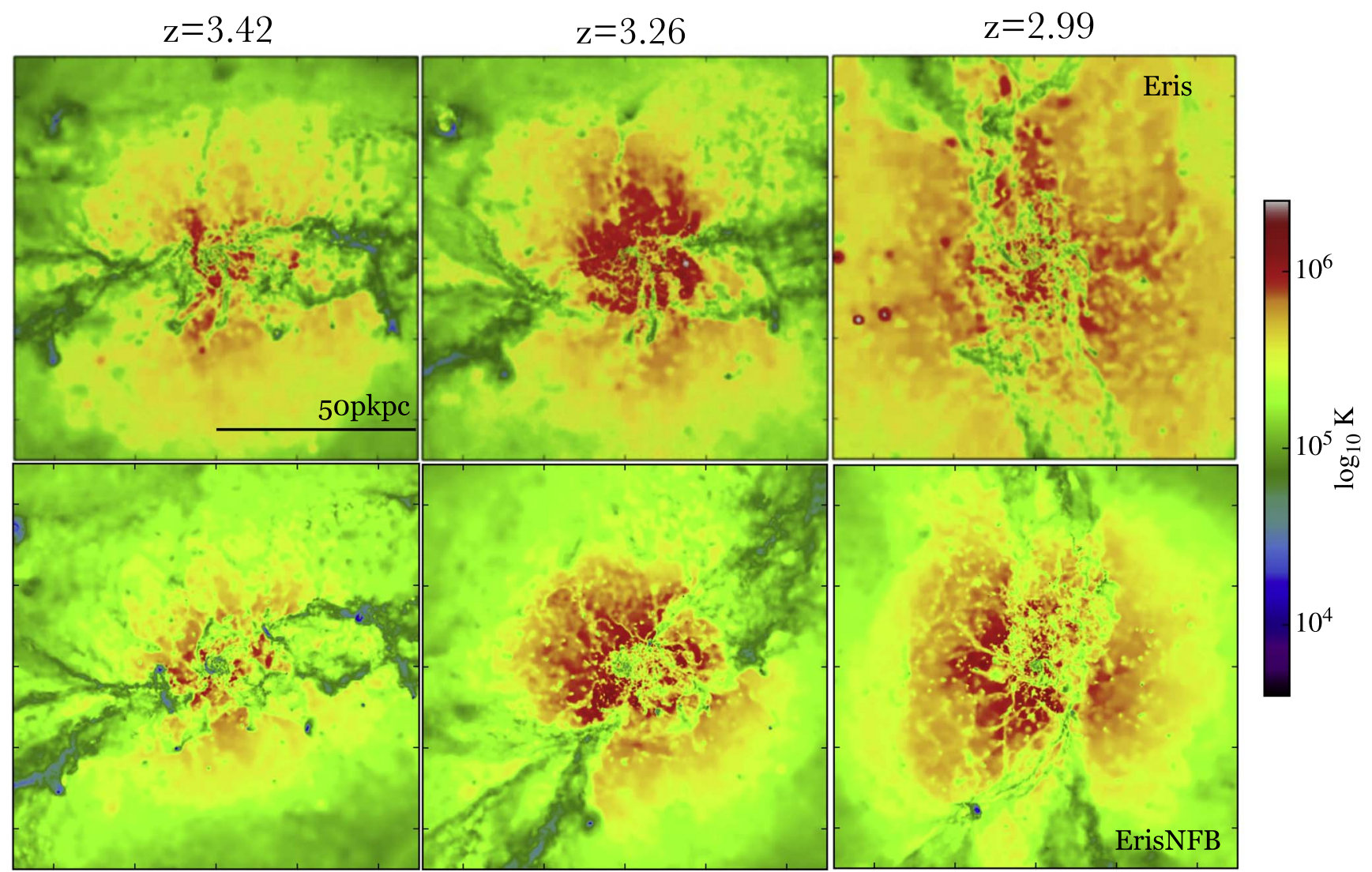

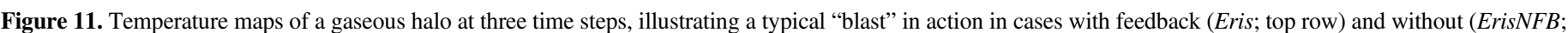

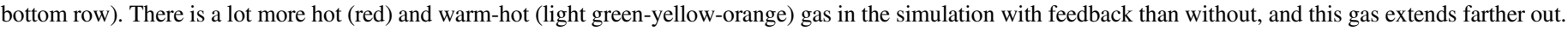
The width of each square is $100 \mathrm{pkpc}$.

galactic outflows on the formation and evolution of the gaseous halos are at odds with the assertions of Fielding et al. (2017). There are a number of fundamental differences between our and their studies that may account for the divergent findings, the most important of which is that the Fielding et al. (2017) study is based on simulations in which the galactic halos are represented by initially spherically symmetric gas profiles designed to capture the low-redshift structure of the systems in an idealized fashion. And although they introduce effects that, over time, break the strict spherical symmetry, the absence of the proper time-dependent cosmological framework for how gas accretion proceeds means that the simulations do not allow for strongly nonlinear spatial asymmetries in the form of mergers and filamentary accretion that arise in and are key features of realistic hierarchical cosmic structure formation simulations, nor do they treat the important effects like the transition from cold to hot mode, etc. As Figure 13 shows, all of these-the different accretion modes and their associated geometries, the mergers, and the galactic outflows triggered by both mergers and stellar feedback-play a pivotal role in the formation and evolution of MW-like halos, and the corresponding symphony (or competition) cannot be neglected even at $z=0$.

\subsection{Corona and the Cold Flows}

As discussed in the Introduction, the galactic halos of MWlike systems occupy a transition mass scale, prior to which the halos are expected to acquire gas primarily via the "cold mode" and thereafter via the "hot mode." In this picture, the gas that enters the halo at early times comes in via cold, dense filamentary streams, remains cold, and ends up flowing onto the central galaxy. As we have demonstrated in this paper, some of this gas is expelled from the galaxy as a result of SNpowered and merger-induced shock heating, thus initiating the inside-out formation of an atmosphere of hot and warm-hot gas in the halo. This happens at an earlier epoch than what was previously established, and in halos of lower mass than the critical mass of $\sim 10^{12} M_{\odot}$, i.e., before the gaseous halo can be established by purely gravitational means. The emergence of this atmosphere sets the stage for a stable accretion shock at the virial radius. Concurrently, on the supragalactic scale, the cosmic filaments are growing, and as their widths become comparable to the sizes of the halos, an increasing fraction of the gas flows into the halos in quasi-spherical fashion, shock heating as it encounters the accretion shock.

There is one outstanding question that we have touched upon previously but have not yet examined, and that is whether the diffuse gas in the halos, and specifically the outflowing gas expelled from the galaxy, affects the galaxy-filaments connection. We briefly discuss this connection in this subsection. A detailed analysis will be presented in a follow-up paper.

During explosive outflow events, while the halo is in the cold mode accretion phase, the gaseous halo can be thought of as comprising two colliding, shearing, and sometimes turbulent flows: cold dense inflowing filamentary streams and powerful hot/warm-hot outflowing wind. The wind generally follows the path of least resistance and streams around and often perpendicular to the filaments. However, in the inner halo, 

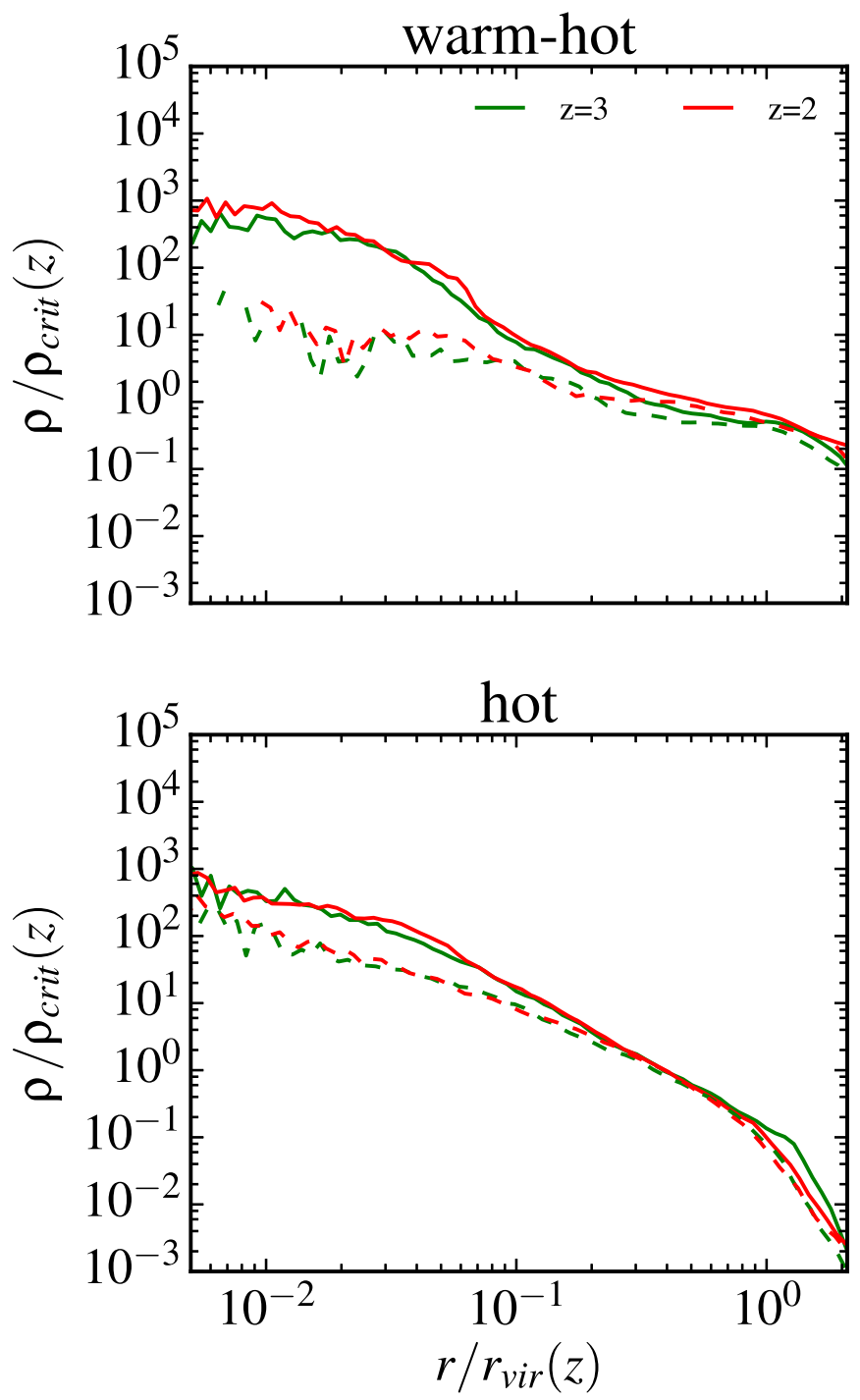

Figure 12. Density profiles of warm-hot (top) and hot (bottom) gas with SN feedback (solid lines) and without (dashed lines). The density and radius are normalized to the critical density of the universe and virial radii of halos at the redshifts under consideration.

two opposing flows do interact strongly and exchange energy and momentum.

To illustrate what happens at these times, we show in Figure 13 snapshots of $p_{\mathrm{wh}} / p_{\mathrm{cf}}$ at four different redshifts. Here, $p_{\mathrm{wh}}$ is the total pressure (i.e., the sum of thermal pressure, $p_{t h}=\rho k T / \mu m_{p}$, and ram pressure, $\left.p_{\text {ram }}=\rho v_{r}^{2} / 2\right)$ of the $T>10^{5} \mathrm{~K}$ gas, and $p_{\mathrm{cf}}$ is the total pressure of the cold inflowing gas, defined as gas with temperatures $T<10^{5} \mathrm{~K}$ and $\vec{v} \cdot \vec{r}<0$. In Figure 13, blue regions correspond to the inflowing streams and red to hot outflowing wind streaming out of the galaxy.

In the absence of powerful outflows, the disk in Eris is fed by two dominant, oppositely oriented streams that drill their way to the center of the halo and connect smoothly to the disk. In Venus, the disk is bombarded by clumps of cold gas from all directions, reflecting the active merging history of this run. Eventually, the inflow in Venus settles down into the same pattern as Eris; however, this happens at $z \approx 1.5$, so we will concentrate on Eris.
At early times (i.e., $z \gtrsim 2$ ), the outflows have a relatively limited impact on the filaments. Looking closely at the center of the $z=3$ and 2 panels, we observe that the blue streams, which ordinarily would converge and terminate on the central galaxy, have been disrupted. The ram pressure of the outflowing wind causes the mouths of the streams to broaden and break up into thin rivulets. However, this delta-like feature remains relatively close to the disk. And once the winds wane, the steady inflow of cold gas along the streams re-establishes the filaments within the inner halo, which then reattach to the central galaxy. However, as the filamentary flow begins to weaken, the total pressure of the outflows is able not only to cause the streams' mouths to fray and broaden but also to disconnect them from the galaxy and push the mouths away to increasingly larger radii. In effect, the winds accelerate the destruction of the streams.

In Figure 14, we compare qualitatively the location and the structure of the filaments in Eris and ErisNFB at five snapshots at redshifts $2 \lesssim z \lesssim 4$ to gauge specifically the impact of SNpowered outflows. The panels show the temperature maps of the gas in a face-on view of the central galaxy, with Eris results presented in the top row and ErisNFB results from the same epochs juxtaposed in the bottom row. The width of each square is 500 comoving Mpc.

Looking first at Eris results (top row), we note that initially the cold streams easily reach and feed the galactic disk of Eris (panel (a)). Powerful outflows fray the mouths of the streams and push them back, often detaching them for extended periods of time from the galaxy (in panel (b), the push-back region is marked by the two dashed lines). Frequently, the filaments manage to maintain connection with the galaxies via many small rivulets (panel (c)), but as the coherence of the streams progressively weakens, they are more easily detached from the galaxy (panels (d) and (e)).

The outflows in ErisNFB (bottom panels) act somewhat similarly but, in this instance, are merger related and are much weaker. Consequently, they do not affect the filaments as strongly as in Eris. Comparing the top and bottom panels in columns (b), (d), and (e), we find that the outflows engendered by mergers and feedback acting in concert (top row) are able to push the mouths of the filaments back further. Also, the merger + feedback-fed outflows and the coronal gas start to heat and strip the filaments as early as $z=3.83$ (see circles in columns (a) and (c)).

In effect, feedback not only helps maintain a larger mass of gas in the hot corona and the warm-hot atmosphere but also erodes the cold flows more effectively and accelerates the breakdown of the direct connection between the streams and the central galaxy. This in turn contributes to the regulation and quenching of star formation in the central galaxies. In effect, our results suggest that although $\mathrm{SN}$-powered outflows are categorized as ejective feedback (i.e., star formation is quenched by expelling the fuel for star formation), in practice they also act to strangle the galaxy by choking off its supply of fresh gas. In other words, SN-powered outflows act as both ejective and preventive feedback.

The above result raises questions about the kinetic "decoupled winds" approach (e.g., Springel \& Hernquist 2003; Oppenheimer \& Davé 2006, 2008; Vogelsberger et al. 2013; Davé et al. 2016; Liang et al. 2016; Pillepich et al. 2018) to modeling stellar feedback. As implemented at present, this approach is not designed to act in a preventative capacity along 


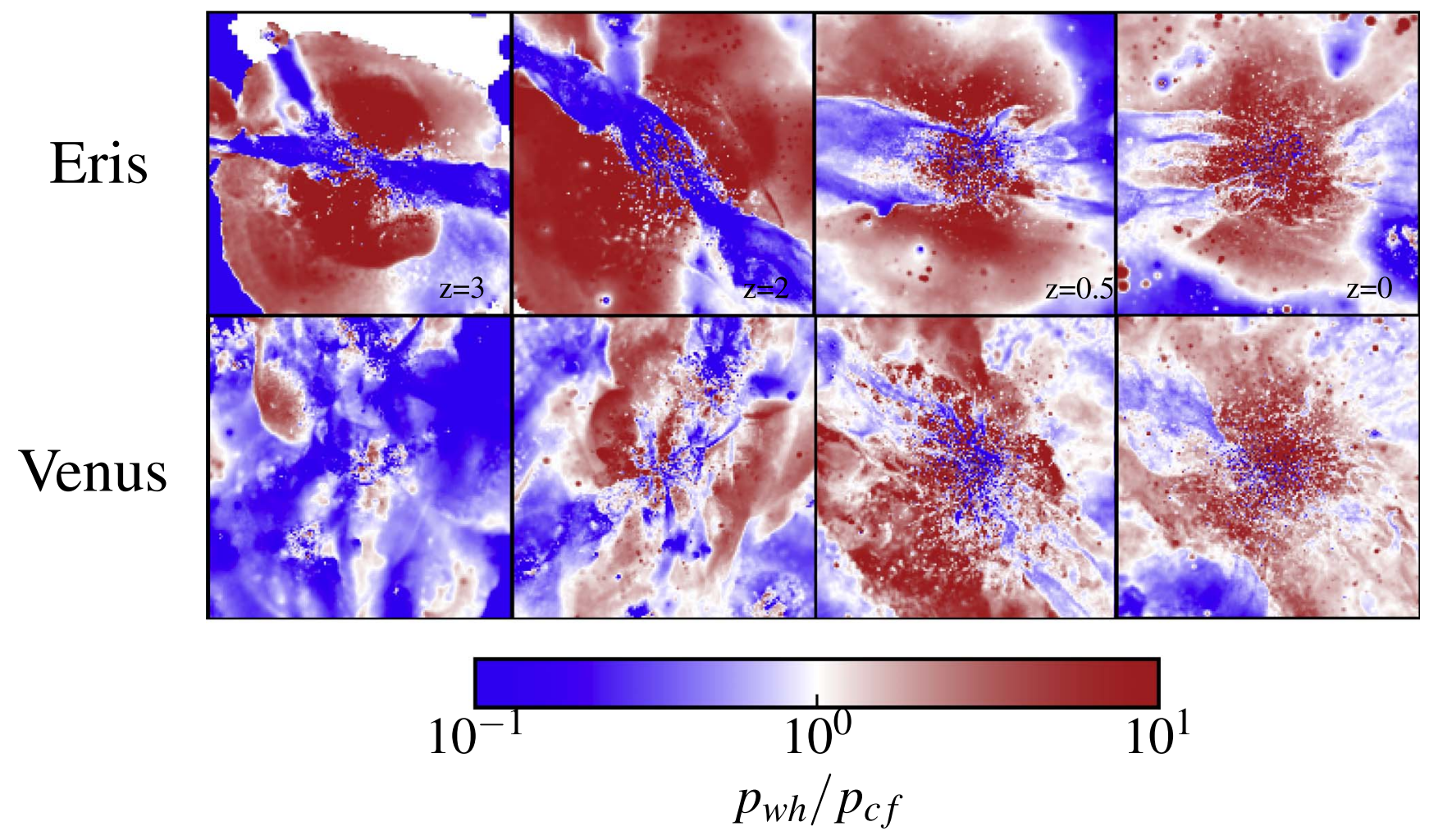

Figure 13. Snapshots of the ratio of the total pressure (i.e., the sum of ram and thermal pressure) of the $>10^{5} \mathrm{~K}$ gas to the total pressure of the $<10^{5} \mathrm{~K}$ inflowing gas, at two redshifts when the cold mode accretion is dominant (two left panels) and at two redshifts when the hot mode acceleration is ascendant (two right panels). The width of each square is $1 \mathrm{pMpc}$.

the lines described above. As such, one would expect that the feedback scheme likely needs to be superejective to achieve the same results. We will return to this issue in future work.

\subsection{Eris $2 k$}

As mentioned in Section 2, Eris and ErisNFB are part of a larger suite of simulations carried out using identical IC where we experiment with the modeling details of the various subgrid physical phenomena, including star formation, cooling, and feedback, in order to see whether we can successfully reproduce the observed stellar mass-halo mass relationship across a range of redshifts (Behroozi et al. 2013; Moster et al. 2018) while still ending up with a late-time spiral galaxy that matches the MW as well as Eris. Here, we briefly discuss the Eris $2 k$ simulation. As described in Section 2, the key defining features of Eris $2 k$ are (i) metal diffusion, (ii) the introduction of the metal-line cooling channel for the $T>10^{4} \mathrm{~K}$ gas, (iii) more efficient cooling of $T<10^{4} \mathrm{~K}$ gas, and (iv) boosted SN feedback (Shen et al. 2012, 2013; Sokołowska et al. 2016, 2017).

Metal-line cooling in Eris $k$ is computed via tables generated using CLOUDY (Ferland et al. 1998) under the assumption that the metals are in ionization equilibrium (Shen et al. 2010) in the presence of an updated cosmic ionizing background (Haardt \& Madau 2012). One could argue that the lack of metal-line cooling in Eris is not self-consistent since the very SN-powered outflows that appear to play a crucial role in the early assembly and evolution of the gaseous corona also continuously introduce metals into the warm-hot and hot circumgalactic medium (CGM). A number of studies (e.g., van de Voort et al. 2011) have shown that the increased efficiency of cooling associated with metal-line radiation results not only in cooler temperatures for the halo gas but also in an increased deposition of gas onto the galaxy. In fact, in the absence of any counteracting mechanism, metal-line cooling can lead to unrealistically high stellar mass.

Additionally, as mentioned in Section 3.1, enhanced cooling also leads to a higher halo mass threshold at which the accretion onto the halos transitions from predominantly cold to predominantly hot mode (and can sustain a stable virial shock; see Figure 3). Van de Voort et al. (2011), who allow for both self-consistent enrichment of the gas and metal cooling in their simulations, find that the threshold mass ranges from $1.6 \times 10^{12} M_{\odot}$ at $z \approx 3$ to $1 \times 10^{12} M_{\odot}$ at $z \approx 2$ to $7 \times 10^{11} M_{\odot}$ at $z \approx 0$. Given their identical mass accretion history, the Eris suite of simulations (i.e., Eris, ErisNFB, Eris $2 k$, etc.) crosses this higher mass threshold at $z \approx 0.3$.

The recipes for star formation and SN feedback in Eris $2 k$ are the same as in Eris and ErisNFB; however, there are some differences in the values of some of the controlling parameters. For instance, the star formation threshold $n_{\mathrm{SF}}$ in Eris $2 k$ is set at 100 atoms $\mathrm{cm}^{-3}$; the maximum temperature of a particle allowed to participate in star formation is $T_{\max }=1 \times 10^{4} \mathrm{~K}$, SN efficiency parameter is increased to $\epsilon_{\mathrm{SN}}=1.0$, and the stellar IMF is updated to Kroupa (2001) (see Table 1 in Section 2 for an overview of the differences between Eris and Eris2k).

The strength of feedback depends on the number of $\mathrm{SNe}$ produced, which in turn is governed globally by the IMF and locally by the star formation density threshold. The revised IMF yields about a factor of 2.8 more SNe for the same star formation rate. Moreover, as explained in detail in Guedes et al. (2011) and Mayer (2012), the local star formation rate, and thus the local impact of $\mathrm{SNe}$, can be boosted significantly by raising 

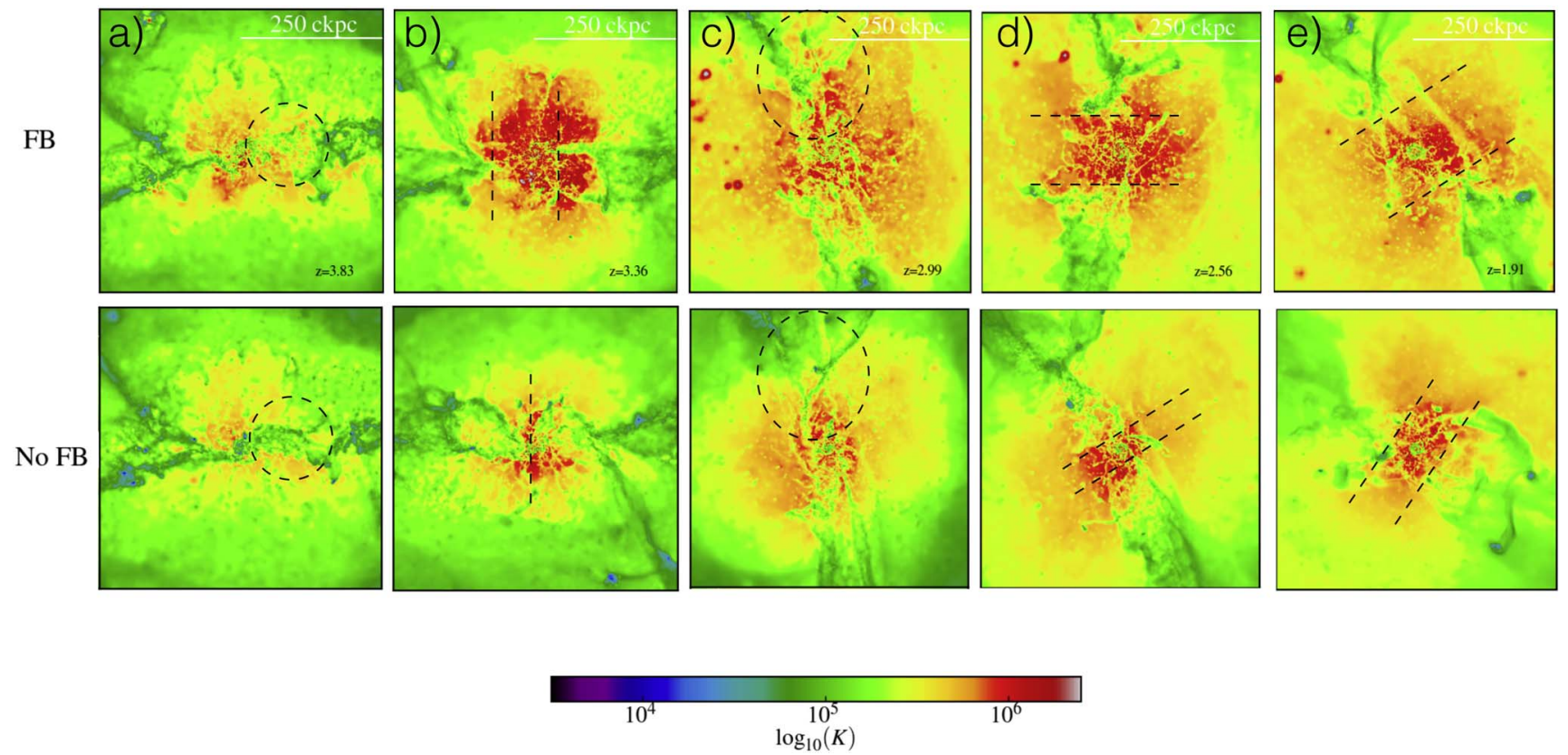

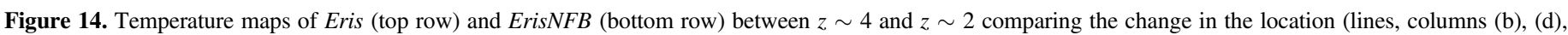
and (e)) and structure (circles, columns (a) and (c)) of the filaments with and without feedback.

the star formation density threshold and allowing the ISM to become more inhomogeneous, an effect that saturates only at very high resolution and density thresholds, well above those resolvable in the current generation of cosmological simulations (Hopkins et al. 2012).

In Eris $2 k$, SN feedback is boosted both globally and locally using parameters that were chosen to achieve realistic stellar masses in accordance with abundance matching at high and low redshift. And indeed, owing to a more effective squelching of star formation at early times, the simulation yields a much better agreement to the Behroozi et al. (2013) stellar mass-halo mass relationship than Eris and its other variants up to $z \sim 0.5$. The stellar mass at earlier epochs is lower than in Eris. At low redshifts, however, Eris $2 k$ deviates significantly from Eris, and the corresponding galactic system is a much less faithful replica of an MW-like late-type spiral (Sokołowska et al. 2017).

In the top panel of Figure 15, we juxtapose the star formation histories of Eris, Venus, and Eris2k. Eris and Venus differ at early times. Eris has a much higher star formation rate until $z=2$; thereafter, it drops steadily over the course of $2 \mathrm{Gyr}$ and stabilizes at $\sim 1-2 M_{\odot} \mathrm{yr}^{-1}$. The star formation in Venus rises more slowly and reaches the peak value later compared to Eris, and the epoch of high star formation is slightly longer. This is due to Venus's late assembly. At late times, however, the star formation settles at a similar rate to that in Eris.

The star formation history in Eris $2 k$ differs from Eris at all epochs, and in this case, this is due to differences in how the subgrid physics unfolds in the two simulations. The boosted feedback in Eris $2 k$ successfully tempers the star formation rate at early times and allows the system to evolve in sync with abundance matching expectations by expelling more gas out of the halo and pushing this gas farther away than in Eris (see Table 3 in Paper I: the fraction of baryons within the virial radius $\left[r_{\text {vir }}\right]$ and $3 r_{\text {vir }}$ is $71 \%$ and $91 \%$ in Eris, compared to $67 \%$ and $74 \%$ in Eris $2 k$ ). This balance among cooling, star formation, and feedback works well until approximately
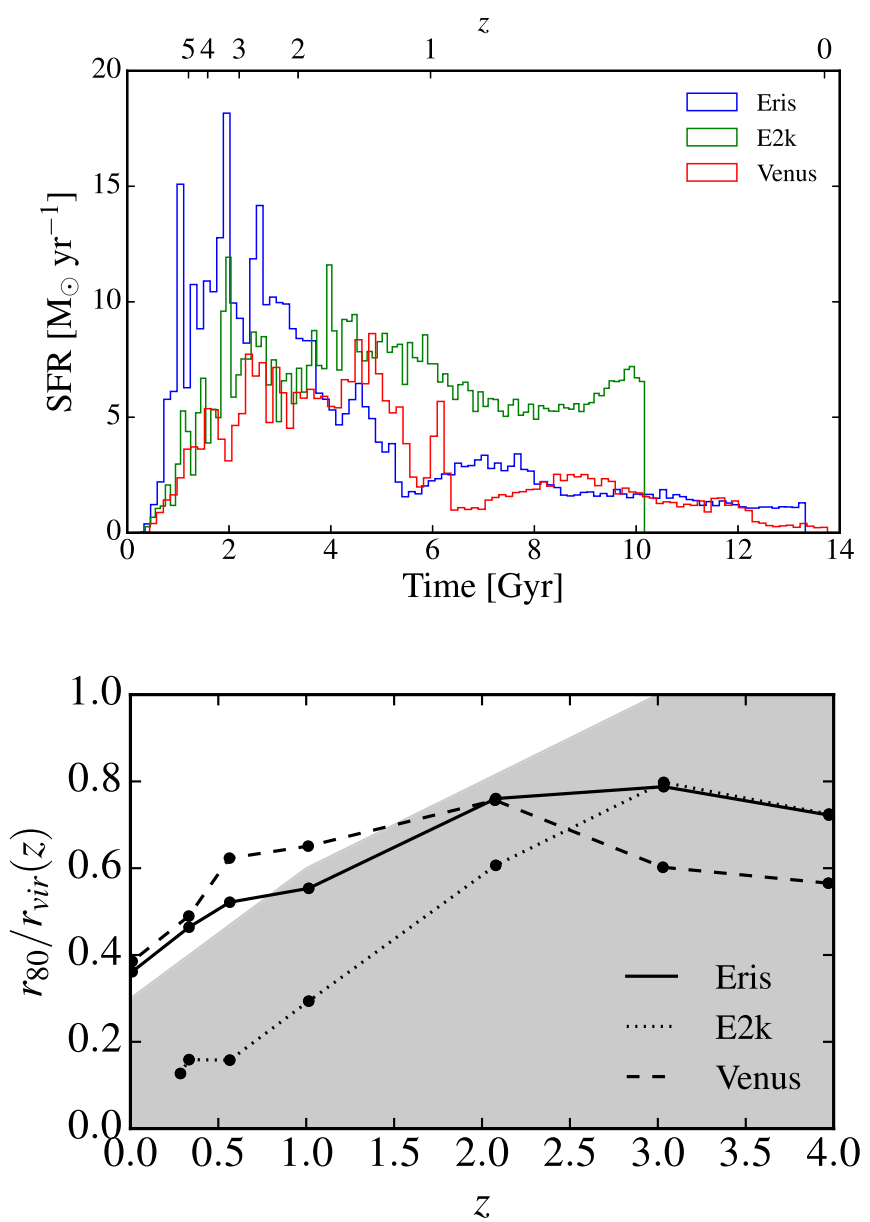

Figure 15. Top panel: comparison of star formation histories of Eris, Eris $2 k$, and Venus. Note that the run Eris $2 k$ was stopped after $10 \mathrm{Gyr}$ of evolution. Bottom panel: radius of a hot corona encompassing $80 \%$ of its mass as a function of redshift. 
$z \approx 0.6$, after which it breaks down. The forming galaxy's deepening potential well increasingly confines the galactic wind within the halo. In the case of Eris $2 k$, the metal-line radiative losses accelerate the cooling of the CGM, and the resulting increased flow of gas onto the galaxy provides fuel for enhanced star formation. Consequently, the already high star formation rate (compared to both Eris and the MW) begins to rise. Overall, Eris $2 k$ converts more gas into stars, and this difference is largely due to late-time star formation: At $z=0.5$, the stellar masses in Eris and Eris $2 k$ differ by only $1 \%$. By $z=0.3$, Eris $2 k$ has $10 \%$ more mass in stars: $M_{*}=3.6 \times$ $10^{10} M_{\odot}$ and $4 \times 10^{10} M_{\odot}$ in Eris and Eris $2 k$, respectively. And given the rising star formation rate in Eris $2 k$, we expect the difference to continue to grow to $z=0 .{ }^{9}$ Additionally, the galaxy in Eris $2 k$ has a kinematically hotter disk (Sokołowska et al. 2017), and as discussed in Paper I, the X-ray luminosity of the Eris $2 k$ system in the $0.5-2 \mathrm{keV}$ band is $L_{\mathrm{X}}=3 \times$ $10^{42} \mathrm{erg} \mathrm{s}^{-1}$, which is 100 times higher than the Eris result, as well as the most current estimates for the MW system.

The bottom panel of Figure 15 illustrates one of the reasons why the X-ray luminosity of Eris $2 k$ is so much higher. The figure shows the evolution of the radius encompassing $80 \%$ of a hot corona in Eris, Venus, and Eris $2 k$ (see Figure 7). Prior to $z=3, r_{80}$ in Eris and Eris $2 k$ evolve in lockstep, but after $z=3$, the $r_{80}$ in Eris $2 k$ begins to shrink and drops to half of the value of $r_{80}$ in Eris. The reduced size of the corona in Eris $2 k$ is not due to there being less mass. The amount of gas in the corona at $z=0.5$ in the two simulations differs by only $10 \%$, with the corona in Eris $2 k$ being the more massive of the two (Sokołowska et al. 2016). The coronal gas distribution in Eris $2 k$ is more compact, and the mean gas density is a factor of $\sim 10$ higher. In addition, as reported in Paper I, the hot corona of Eris $2 k$ has significantly higher metallicity that, even at comparable densities, results in increased X-ray luminosity via line emissions, as well as boosted bremsstrahlung radiation. The combination of higher density and higher metallicity explains why the X-ray luminosity of the system is a factor of 100 larger than in the other simulations. The enhanced cooling also results in more robust, thicker cool-gas filaments that are more resilient to disruption.

That Eris $2 k$ fails to match the properties of the MW system (i.e., the galaxy and its gaseous halo) highlights potential concerns with the current treatment of metal-line cooling (see Christensen et al. 2014; Tremmel et al. 2018). One possible problem is that metal-line cooling is computed under the assumption that the ions are in ionization equilibrium, which can lead to an overestimate of the cooling rate if the ions are not in equilibrium. Oppenheimer \& Schaye (2013) show that the warm-hot component of the CGM is most susceptible to this, and the effect is even stronger if the gas is subject to fluctuating ionizing radiation from an active galactic nucleus (Oppenheimer \& Schaye 2013). It is also highly likely that the lack of heating sources, such as compact X-ray binaries (Madau \& Fragos 2017), cosmic-ray heating, photoelectric heating, etc., in our simulations may also be a contributing factor. Also, we do not account for the effects of local ionization radiation, which can also alter the cooling profile of the CGM. Additionally, Christensen et al. (2014) (see also Tremmel et al. 2018) have shown that the inclusion of metal cooling in simulations that do not have sufficiently high

\footnotetext{
9 We note that the Eris $2 k$ simulation had to be stopped at 10 Gyr owing to the exhaustion of computational resources.
}

resolution to support the proper modeling of molecular hydrogen physics and the multiphase structure of the ISM and the CGM results in overcooling. In simulations that attempt to do so, they find that the resulting galaxies have star formation histories and outflow rates that are more similar to those in the primordial cooling runs (like our Eris) than to those that include metal-line cooling but have inadequate resolution (like Eris $2 k$ ).

In summary, the combination of boosted feedback and metal cooling, particularly, has a significant impact on both the evolution of the resulting galactic system, including the structure of the galactic disk and the corona, and the ability of SN-driven shocks to disrupt the cold filaments feeding the galaxies. However, given the various concerns about the modeling of metal cooling in simulations at the present time, it is not clear whether Eris $2 k$ is necessarily a better representation of reality. In fact, running without metal cooling may well be preferable, as the approach appears to better approximate the dynamics and thermodynamics of gas components with moderate to high average densities.

\section{Conclusions}

We use a set of four hydrodynamical zoom-in simulations of MW-like galactic systems-described in Section 2-to study the halo-filling gaseous atmosphere cocooning the central galaxy. We analyzed the various properties of the gaseous halos in our simulated systems in Paper I (Sokołowska et al. 2016) and compared these against available observational results for the MW. In the present paper, we investigate the mechanisms that contribute to the formation of the gaseous halo.

The conventional view is that the gaseous atmosphere arises when the cosmological influx of gas onto a galactic halo transitions to diffuse quasi-spherical inflow. This gas is heated to near-virial temperatures by accretion shocks and in the interior of the halos is heated further owing to compression as long as radiative cooling is relatively inefficient. While this picture is valid in the sense that the late-time mass budget of the gaseous halo is generally dominated by gas accreted in this fashion, the details of how the gaseous halos arise are considerably more involved. We find-and this is our most important result - that the gaseous halo and especially the corona $^{10}$ are not simply the products of hot spherical accretion. We trace their origin to gas expelled from the galaxy by merger-induced shock heating and SN feedback. This is why they are present in our simulations much earlier than expected (as early as $z \approx 3-4$ ) and can be seen even around lower-mass (e.g., $M_{\text {halo }} \lesssim 10^{11} M_{\odot}$ ) halos.

In detail, our simulations show that prior to $z \approx 2$, most of the gas composing the halo-filling warm-hot atmosphere is gas that was expelled from the central galaxy and cooled as it expanded outward. Only after $z \approx 2$ does this inside-out process become inverted. Moreover, we find that direct SN heating is the dominant heating mode for the warm-hot gas at all redshifts.

The corona, which comprises gas with temperatures a factor of 2-2.5 greater than the virial temperature of the system at $z=0$, is of particular interest because, although it is not the

\footnotetext{
${ }^{10}$ We categorize the gas composing the gaseous halo into two components: the hot $\left(T>10^{6} \mathrm{~K}\right) \mathrm{X}$-ray-luminous corona, and the dominant (in terms of mass) warm-hot $\left(T=10^{5}-10^{6} \mathrm{~K}\right)$ component.
} 
most abundant component in terms of mass, it is the phase that is primarily responsible for the diffuse X-ray emission and for $\mathrm{O}$ VII/O VIII emission/absorption observed in the halo. In our simulations, the coronal gas is centrally concentrated and makes up nearly a constant fraction $(13 \%-15 \%)$ of the gaseous atmosphere in the primary halo over the redshift range $z \lesssim 2.5$, regardless of whether the primary halo has formed or is still in the throes of active formation. We also showed that a significant fraction of the gas that makes up the $z=0$ corona entered the halo via cold filamentary streams. It becomes part of the corona only after being heated to temperatures in excess of $10^{6} \mathrm{~K}$ by $\mathrm{SN}$ heating and merger-induced shocks and expelled from the central galaxy.

That mergers play a role in generating the warm-hot/hot medium around disk galaxies has been previously seen in idealized, noncosmological simulations of merging galaxies (Cox et al. 2006; Sinha \& Holley-Bockelmann 2009). The present study confirms that this also happens in galactic systems forming within a realistic cosmological setting. Further, the impact of the merger-induced outflows is much more pronounced when $\mathrm{SN}$ feedback is acting in concert. With respect to the latter, we note that large-scale SN-powered galactic winds are a crucial feature of the contemporary galaxy formation models, without which it is not possible to obtain realistic galaxy properties across the desired range of mass scales and galaxy types (e.g., Christensen et al. 2016; Davé et al. 2016; Garrison-Kimmel et al. 2017; Pillepich et al. 2018; see also Somerville \& Davé 2015 and references therein).

The wind-fed corona and the winds themselves play a pivotal role in regulating the flow of gas from the cosmic web to the galaxy. In the absence of thermal winds, the filaments funnel cold gas onto the galaxy until the evolution of the largescale structure causes the filaments to thicken and the gas accretion model to become diffuse and quasi-spherical. We find that powerful galactic winds can disrupt the filament-galaxy connection well before the cosmological transition happens, repeatedly detaching the galaxy from its filamentary network and even accelerating the destruction of the connection altogether. In other words, the winds and the corresponding corona take on a preventive role, in addition to their wellestablished ejective role. This is an intriguing twist with profound implications. For one, it would mean that all "bathtub-type" galaxy formation models, a category that includes most of the numerical simulations that use the decoupled kinetic wind prescription (see Somerville \& Davé 2015, for a full list of simulation studies that model galactic winds in this fashion), are inadequate because they focus exclusively on the ejective aspect of the galactic winds. In this class of models, galaxy evolution is governed primarily by the competition between gas accretion and expulsion, and the mass-loss rate is tied to the star formation rate (Bouché et al. 2010; Lilly et al. 2013). In light of our findings, we have initiated a much more thorough analysis of galactic winds as "agents" of both ejective and preventative feedback.

Finally, apart from the above puzzle about the action of galactic winds, our study leaves unresolved several other issues. For instance, given our small number of simulations and the restricted choice of subgrid feedback models adopted, one could wonder how our key findings would have changed had we considered a much larger sample of IC, as well as subgrid recipes for feedback. One thing is clear, though. The assembly history of the galaxy is an important factor, especially since the corona appears to act in a preventive feedback capacity and mergers play a crucial role in generating the hot corona at early times by triggering both shock-heated outflows and starbursts that further boost the outflows. It then follows that the number of major mergers occurring before the halo has grown enough to sustain a stable shock may be important in determining when a spiral galaxy begins to quench. We may have already seen a hint of this within our simulation set: the growth of the hot corona in Venus, which has a much more active merging history, departs considerably from the predictions of the standard accretion shock picture when compared to Eris. We speculate that for systems of comparable final mass, perhaps quenching is more effective and starts sooner in spiral galaxy systems with active merging history. If borne out, this could be a major new addition to the current paradigm for galaxy formation and could, for example, help explain the scatter in the stellar-to-halo mass relationship in the vicinity of the MW mass scale (see Moster et al. 2018). This is yet another aspect to be investigated.

The authors would like to thank Simon White, Andrey Kravtsov, Crystal Martin, Jerry Ostriker, Joop Schaye, Ali Rahmati, Sebastian Trujillo-Gomez, Mike Fall, Neal Katz, and Carlos Frenk for valuable discussions that contributed to this paper. L.M. thanks the Kavli Institute for Theoretical Physics at UC Santa Barbara for hospitality during the "Galaxy-Halo Connection" Program in Spring 2017, during which preliminary results of this work were presented and discussed. A.B. acknowledges support from NSERC (Canada) through the Discovery Grant program and from the Pauli Center for Theoretical Studies ETH UZH and is grateful to the University of Zurich's Institute for Computational Sciences, and especially the members of the Institute's Center for Theoretical Astrophysics and Cosmology, for hospitality during his extended visits in winter 2016 and spring 2017.

\section{ORCID iDs}

A. Sokołowska (iD https://orcid.org/0000-0003-0898-8247

A. Babul (i) https://orcid.org/0000-0003-1746-9529

S. Shen (1) https://orcid.org/0000-0001-8523-1171

P. Madau (i) https://orcid.org/0000-0002-6336-3293

\section{References}

Agertz, O., \& Kravtsov, A. V. 2015, ApJ, 804, 18

Anders, E., \& Grevesse, N. 1989, GeCoA, 53, 197

Anderson, M. E., \& Bregman, J. N. 2011, ApJ, 737, 22

Anderson, M. E., Churazov, E., \& Bregman, J. N. 2016, MNRAS, 455, 227

Babul, A., \& Rees, M. J. 1992, MNRAS, 255, 346

Balogh, M. L., Babul, A., \& Patton, D. R. 1999, MNRAS, 307, 463 Behroozi, P. S., Wechsler, R. H., \& Conroy, C. 2013, ApJ, 770, 57

Binney, J. 1977, ApJ, 215, 483

Birnboim, Y., \& Dekel, A. 2003, MNRAS, 345, 349

Bogdán, Á, Forman, W. R., Kraft, R. P., \& Jones, C. 2013a, ApJ, 772, 98 Bogdán, Á, Forman, W. R., Vogelsberger, M., et al. 2013b, ApJ, 772, 97

Bouché, N., Dekel, A., Genzel, R., et al. 2010, ApJ, 718, 1001

Bower, R. G., Benson, A. J., \& Crain, R. A. 2012, MNRAS, 422, 2816

Bromm, V., Ferrara, A., Coppi, P., \& Larson, R. B. 2001, MNRAS, 328, 958

Brooks, A. M., Governato, F., Quinn, T., Brook, C. B., \& Wadsley, J. 2009, ApJ, 694, 396

Cautun, M., van de Weygaert, R., Jones, B. J. T., \& Frenk, C. S. 2014, MNRAS, 441, 2923

Christensen, C. R., Davé, R., Governato, F., et al. 2016, ApJ, 824, 57

Christensen, C. R., Governato, F., Quinn, T., et al. 2014, MNRAS, 440, 2843

Cox, T. J., Di Matteo, T., Hernquist, L., et al. 2006, ApJ, 643, 692

Davé, R., Finlator, K., \& Oppenheimer, B. D. 2012, MNRAS, 421, 98 
Davé, R., Oppenheimer, B. D., \& Finlator, K. 2011, MNRAS, 415, 11 Davé, R., Thompson, R., \& Hopkins, P. F. 2016, MNRAS, 462, 3265 Dekel, A., \& Birnboim, Y. 2006, MNRAS, 368, 2

Dekel, A., Birnboim, Y., Engel, G., et al. 2009, Natur, 457, 451

Dekel, A., \& Silk, J. 1986, ApJ, 303, 39

Fang, T., Buote, D. A., Bullock, J. S., \& Ma, R. 2015, ApJS, 217, 21

Fardal, M. A., Katz, N., Gardner, J. P., et al. 2001, ApJ, 562, 605

Ferland, G. J., Korista, K., Verner, D., et al. 1998, PASP, 110, 761

Fiacconi, D., Feldmann, R., \& Mayer, L. 2014, MNRAS, 446, 1957

Fielding, D., Quataert, E., McCourt, M., \& Thompson, T. A. 2017, MNRAS, 466, 3810

Garrison-Kimmel, S., Hopkins, P. F., Wetzel, A., et al. 2017, arXiv:1712.03966

Guedes, J., Callegari, S., Madau, P., \& Mayer, L. 2011, ApJ, 742, 76

Gupta, A., Galeazzi, M., Koutroumpa, D., Smith, R., \& Lallement, R. 2009, ApJ, 707, 644

Gupta, A., Mathur, S., Krongold, Y., Nicastro, F., \& Galeazzi, M. 2012, ApJL, 756, L8

Haardt, F., \& Madau, P. 1996, ApJ, 461, 20

Haardt, F., \& Madau, P. 2012, ApJ, 746, 125

Henley, D. B., \& Shelton, R. L. 2012, ApJS, 202, 14

Henley, D. B., \& Shelton, R. L. 2013, ApJ, 773, 92

Hopkins, P. F., Quataert, E., \& Murray, N. 2012, MNRAS, 421, 3488

Joung, M. R., Putman, M. E., Bryan, G. L., Fernández, X., \& Peek, J. E. G. 2012, ApJ, 759, 137

Katz, N., Keres, D., Dave, R., \& Weinberg, D. H. 2003, ASSL, 281, 185

Kereš, D., Katz, N., Fardal, M., Davé, R., \& Weinberg, D. H. 2009, MNRAS, 395, 160

Kereš, D., Katz, N., Weinberg, D. H., \& Davé, R. 2005, MNRAS, 363, 2

Kroupa, P. 2001, MNRAS, 322, 231

Kroupa, P., Tout, C. A., \& Gilmore, G. 1993, MNRAS, 262, 545

Larson, R. B. 1974, MNRAS, 169, 229

Lewis, G. F., Babul, A., Katz, N., et al. 2000, ApJ, 536, 623

Liang, L., Durier, F., Babul, A., et al. 2016, MNRAS, 456, 4266

Lilly, S. J., Carollo, C. M., Pipino, A., Renzini, A., \& Peng, Y. 2013, ApJ, 772, 119

Madau, P., \& Fragos, T. 2017, ApJ, 840, 39

Marinacci, F., Pakmor, R., \& Springel, V. 2014, MNRAS, 437, 1750

Mashchenko, S., Wadsley, J., \& Couchman, H. M. P. 2007, Sci, 319, 174

Mathews, W. G., \& Baker, J. C. 1971, ApJ, 170, 241

Mayer, L. 2012, in ASP Conf. Ser. 453, Advances in Computational Astrophysics: Methods, Tools, and Outcome, ed. R. Capuzzo-Dolcetta, M. Limongi, \& A. Tornambè (San Francisco, CA: ASP), 289

McKee, C. F., \& Ostriker, J. P. 1977, ApJ, 218, 148

Miller, M., \& Bregman, J. 2013, ApJ, 770, 118

Miller, M. J., \& Bregman, J. N. 2015, ApJ, 800, 14
Moster, B. P., Naab, T., \& White, S. D. M. 2018, MNRAS, 477, 1822

Oppenheimer, B. D., \& Davé, R. 2006, MNRAS, 373, 1265

Oppenheimer, B. D., \& Davé, R. 2008, MNRAS, 387, 577

Oppenheimer, B. D., \& Schaye, J. 2013, MNRAS, 434, 1043

Papovich, C., Labbé, I., Quadri, R., et al. 2015, ApJ, 803, 26

Peeples, M. S., Werk, J. K., Tumlinson, J., et al. 2014, ApJ, 786, 54

Pillepich, A., Springel, V., Nelson, D., et al. 2018, MNRAS, 473, 4077

Poole, G. B., Babul, A., McCarthy, I. G., Sanderson, A. J. R., \& Fardal, M. A. 2008, MNRAS, 391, 1163

Poole, G. B., Fardal, M. A., Babul, A., et al. 2006, MNRAS, 373, 881

Puchwein, E., \& Springel, V. 2013, MNRAS, 428, 2966

Putman, M. E., Peek, J. E. G., \& Joung, M. R. 2012, ARA\&A, 50, 491

Rahmati, A., Schaye, J., Crain, R. A., et al. 2016, MNRAS, 459, 310

Rees, M. J., \& Ostriker, J. P. 1977, MNRAS, 179, 541

Roškar, R., Teyssier, R., Agertz, O., Wetzstein, M., \& Moore, B. 2014, MNRAS, 444, 2837

Sedov, L. I. 1959, Similarity and Dimensional Methods in Mechanics

Shen, S., Madau, P., Aguirre, A., et al. 2012, ApJ, 760, 50

Shen, S., Madau, P., Conroy, C., Governato, F., \& Mayer, L. 2014, ApJ, 792, 99

Shen, S., Madau, P., Guedes, J., et al. 2013, ApJ, 765, 89

Shen, S., Wadsley, J., \& Stinson, G. 2010, MNRAS, 407, 1581

Silk, J. 1977, ApJ, 211, 638

Sinha, M., \& Holley-Bockelmann, K. 2009, MNRAS, 397, 190

Smith, R. K., Brickhouse, N. S., Liedahl, D. A., \& Raymond, J. C. 2001, ApJL, 556, L91

Sokołowska, A., Capelo, P. R., Fall, S. M., et al. 2017, ApJ, 835, 289

Sokołowska, A., Mayer, L., Babul, A., Madau, P., \& Shen, S. 2016, ApJ, 819,21

Somerville, R. S., \& Davé, R. 2015, ARA\&A, 53, 51

Springel, V., \& Hernquist, L. 2003, MNRAS, 339, 289

Stewart, K. R., Maller, A. H., Oñorbe, J., et al. 2017, ApJ, 843, 47

Stinson, G., Seth, A., Katz, N., et al. 2006, MNRAS, 373, 1074

Taylor, G. 1950, RSPSA, 201, 159

Toft, S., Rasmussen, J., Sommer-Larsen, J., \& Pedersen, K. 2002, MNRAS, 335,799

Tremmel, M., Quinn, T. R., Ricarte, A., et al. 2018, arXiv:1806.01282

Tumlinson, J., Thom, C., Werk, J. K., et al. 2011, Sci, 334, 948

van de Voort, F., Quataert, E., Hopkins, P. F., et al. 2016, MNRAS, 463, 4533

van de Voort, F., Schaye, J., Booth, C. M., Haas, M. R., \& Dalla Vecchia, C. 2011, MNRAS, 414, 2458

Vogelsberger, M., Genel, S., Sijacki, D., et al. 2013, MNRAS, 436, 3031

Wadsley, J., Stadel, J., \& Quinn, T. 2004, NewA, 9, 137

White, S. D. M., \& Frenk, C. S. 1991, ApJ, 379, 52

White, S. D. M., \& Rees, M. J. 1978, MNRAS, 183, 341 